\title{
Dual-Resonator-Based (DRB) and Multiple-Resonator-Based (MRB) MEMS Sensors: A Review
}

\author{
Yusi Zhu ${ }^{1,2}$, Zhan Zhao ${ }^{1,2}$, Zhen Fang ${ }^{1,2}$ and Lidong Du $1,2, * \mathbb{D}$ \\ 1 State Key Laboratory of Transducer Technology, Aerospace Information Research Institute, Chinese Academy \\ of Sciences, Beijing 100190, China; zhuyusi16@mails.ucas.ac.cn (Y.Z.); zhaozhan@mail.ie.ac.cn (Z.Z.); \\ zfang@mail.ie.ac.cn (Z.F.) \\ 2 School of Electronic, Electrical and Communication Engineering, University of Chinese Academy of Sciences, \\ Beijing 100049, China \\ * Correspondence: lddu@mail.ie.ac.cn; Tel.: +86-13811347695
}

check for updates

Citation: Zhu, Y.; Zhao, Z.; Fang, Z.; $\mathrm{Du}, \mathrm{L}$. Dual-Resonator-Based (DRB) and Multiple-Resonator-Based (MRB) MEMS Sensors: A Review. Micromachines 2021, 12, 1361. https:/ / doi.org/10.3390/mi12111361

Academic Editors: Amal Z. Hajjaj, Nizar Jaber and Aiqun Liu

Received: 7 September 2021

Accepted: 27 October 2021

Published: 4 November 2021

Publisher's Note: MDPI stays neutral with regard to jurisdictional claims in published maps and institutional affiliations.

Copyright: (c) 2021 by the authors. Licensee MDPI, Basel, Switzerland. This article is an open access article distributed under the terms and conditions of the Creative Commons Attribution (CC BY) license (https:// creativecommons.org/licenses/by/ $4.0 /)$.

\begin{abstract}
Single-resonator-based (SRB) sensors have thrived in many sensing applications. However, they cannot meet the high-sensitivity requirement of future high-end markets such as ultra-small mass sensors and ultra-low accelerometers, and are vulnerable to environmental influences. It is fortunate that the integration of dual or multiple resonators into a sensor has become an effective way to solve such issues. Studies have shown that dual-resonator-based (DRB) and multiple-resonatorbased (MRB) MEMS sensors have the ability to reject environmental influences, and their sensitivity is tens or hundreds of times that of SRB sensors. Hence, it is worth understanding the state-ofthe-art technology behind DRB and MRB MEMS sensors to promote their application in future high-end markets.
\end{abstract}

Keywords: dual-resonator-based (DRB) MEMS sensor; multiple-resonator-based (MRB) MEMS sensor; strength-coupled-resonator-based (SCRB) sensor; wave-coupled-resonator-based (WCRB) sensor; uncoupled-resonator-based (UCRB) sensor

\section{Introduction}

In the past two decades, single-resonator-based (SRB) Micro-Electro-MechanicalSystems (MEMS) sensors have been extensively studied for detecting small physical or chemical perturbations, such as pressure [1-8], rotational rate [9-14], acceleration [15-17], vibration [18-20], force [21-23], chemical vapor [24-26], and biological material [27-34]. Various structures, such as flexural mode resonators (FMR) $[28,31,35]$, surface acoustic wave resonators (SAW) [36], bulk acoustic resonators (BAR) [6,12,24-26,37], lamb wave resonators (LWR) $[8,14,38]$, etc., have been fabricated to enable these SRB sensors to have an excellent performance. A key attribute of these sensors is that the output signal is the variation/shift in the resonant frequency $(\Delta \mathrm{f})$ of a vibrating structure that is subjected to small perturbations in the structural parameters i.e., effective stiffness or effective mass. Additional advantages of this method of detection are simple mechanical design, quasi-digital nature of the signal (thus using simple frequency measurement system such as frequency counter and not requiring additional analog-to-digital (A/D) conversion circuit), and ultra-high resolution [39-41], (up to 10-15 g scale [42-44] and up to 10-18 g scale [45-48]). Accurate frequency references, such as crystal oscillators, offer better stability than typical voltage or current references. Moreover, the output signal frequency of the resonant sensor is considered to be relatively immune to noise and interference [49]. However, they still inevitably suffer from environmental influences [50,51] and cannot satisfy the high-sensitivity requirement of future high-end markets such as ultra-small mass sensors [45] and ultra-low accelerometers [52]. It is fortunate that dual-resonatorbased (DRB) and multiple-resonator-based (MRB) MEMS sensors have become an effective way to solve such issues due to their ability of sensitivity enhancement and rejection of environmental influences. 
Nowadays, DRB and MRB MEMS sensors have attracted great attention from many researchers due to their advantages. Based on the relationship of resonators, DRB and MRB MEMS sensors can be categorized into three classes which include strength-coupledresonator-based (SCRB) sensors [53-108], wave-coupled-resonator-based (WCRB) sensors [109-111] and uncoupled-resonator-based (URB) sensors [112-122].

The first kind of DRB and MRB MEMS sensors are the SCRB sensors, whose resonators are mutually coupled by coupling strength. In fact, the coupling strength can be exerted by mechanical structure, electrostatic force, and magnetic force. According to these coupling methods, SCRB sensors can be further classified into three types. The first type is a mechanical-strength-coupled-resonator-based (ME-SCRB) sensor. One of the advantages is that no further action is needed to couple those resonators together after the fabrication processes. The second type is an electrical-strength-coupled-resonator-based (EL-SCRB) sensor, of which the biggest merit is that the coupling strength can be easily tuned. The third type is a magnetic-strength-coupled-resonator-based (MA-SCRB) sensor, of which the virtue is that the coupling is not limited by the separation distance of those resonators. In recent ten years, those SCRB sensors have been comprehensively studied. Results have shown that strength-coupled resonators enable the SCRB sensors to function with merits of high sensitivity and high environmental stability.

The second kind of DRB and MRB MEMS sensors are the WCRB sensors, of which resonators are acoustically coupled. Those resonators can be thought of as acoustic waveguides, where various acoustic waves, such as bulk acoustic waves, shear horizontal waves, surface acoustic waves and lamb waves can propagate. A variety of resonant modes can be generated by the propagation and reflection of guided waves in the resonator. Therefore, acoustic coupling can be formed by properly adjusting the structure of resonators. Moreover, higher sensitivity can be obtained by precisely measuring the beat frequency. In addition, some WCRB sensors also have the properties of low thermal expansion coefficient of frequency (TCF) due to those low TCF resonant modes and are not easily affected by external temperature interference.

The last kind of DRB and MRB MEMS sensors are the so-called UCRB sensors, of which resonators are not coupled. Basically, they can be divided into three types based on the spatial relationship of resonators. The first type can be named as the DC-UCRB sensor because the resonators are directly connected. Higher sensitivity can be obtained by controlling the beat frequency to a small value. The second type is the dual-mode SRB sensor. It can be termed as quasi-UCRB (Q-UCRB) sensor due to the capability of simultaneously working on dual resonant modes. This mechanism of operation enables the Q-UCRB sensor to work with high sensitivity and low TCF properties. The last type can be called as PS-UCRB sensor, whose resonators are put together but physically separated. The frequencies of resonators will drift in the same or inverse direction in response to temperature changes when putting them in the same environment. Additionally, its drifts can be suppressed by the output of resonators with feasible algorithm or hardware.

In a word, DRB and MRB MEMS sensors have the capabilities of sensitivity enhancement and environmental influences rejection. In view of the above advantages, it is worthwhile to summarize these DRB and MRB sensors to provide reference and guidance for future high-end market applications. With that purpose, we reviewed the-state-of-art of DRB and MRB MEMS sensors as follows: firstly, we analyzed single resonator and coupled resonator; secondly, a detailed introduction and analysis of DRB and MRB sensors, including SCRB sensors, WCRB sensors and UCRB sensors is given; finally, the future development of these sensors is prospected.

\section{Analysis of Resonators}

2.1. Single Resonator

The model of single resonator is shown in Figure 1a. The motion equation for such system can be denoted as:

$$
M_{\mathrm{eff}} \ddot{x}+b_{\mathrm{eff}} \dot{x}+k_{\mathrm{eff}} x=F
$$


where $M_{\text {eff }}, b_{\text {eff }}, k_{\text {eff }}, F$, and $x$ are the equivalent effective mass, effective damping constant, effective spring constant, the applied external force, and the motion displacement, respectively. Under ideal conditions, the frequency can be calculated by:

$$
f=\frac{1}{2 \pi} \sqrt{\frac{k_{\text {eff }}}{M_{\text {eff }}}}
$$

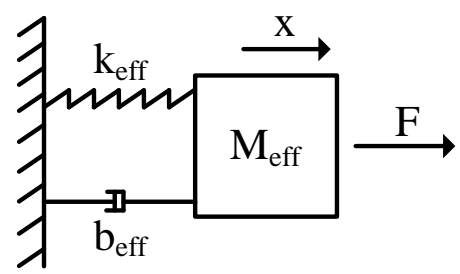

(a)

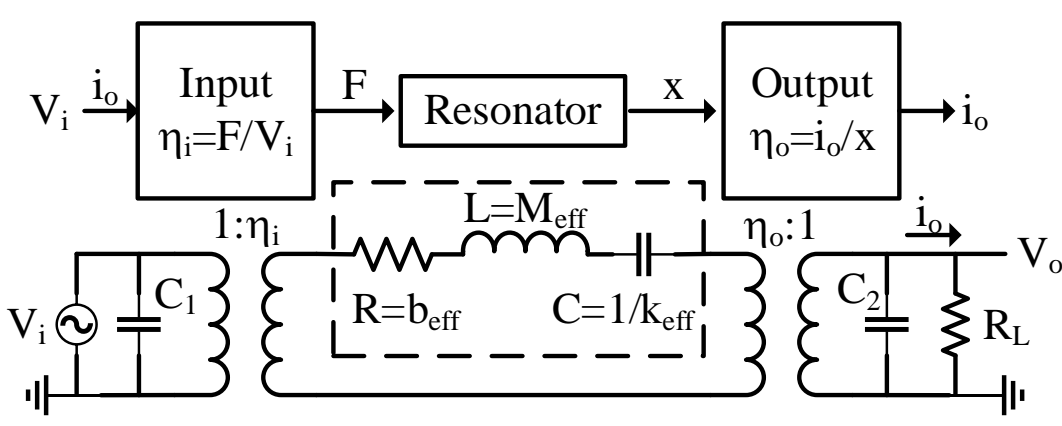

(b)

Figure 1. (a) A simple resonator model; (b) the block diagram and the electrical circuit of a resonator.

Usually, a single resonator can be used to detect perturbation by the change in effective spring constant or/and mass. The relationship between frequency change and perturbation can be established on the basis of Equations (1) and (2). The normalized sensitivity can be obtained by [123]:

$$
\begin{aligned}
& \left|S_{M}\right|=\left|\frac{\partial \omega_{0}}{\partial M} \times \frac{M}{\omega_{0}}\right| \approx \frac{1}{2} \\
& \left|S_{K}\right|=\left|\frac{\partial \omega_{0}}{\partial K} \times \frac{K}{\omega_{0}}\right| \approx \frac{1}{2}
\end{aligned}
$$

However, a single resonator usually suffers from environmental influences, such as temperature, humidity, and pressure, which will result in unwanted shift of resonance frequency, calculated by [123]:

$$
\Delta \omega=\frac{\Delta K+\Delta K_{\text {environment }}}{2 K} \times \omega_{0}
$$

It is obvious that the unwanted frequency shift caused by environmental disturbance will greatly affect the performance of resonator. This disadvantage can be reduced by integrating dual or multiple resonators into a MEMS sensor, because all resonators have the same variation (common change) under the same environmental influences. In addition, the common change can be eliminated by appropriate algorithms or structure during the deduction of the sensor output.

The lumped element model is another method that can be used to analyze and simulate a single resonant system [124,125]. The damping (b), mass (M) and compliance (1/k) of each resonator in the mechanical domain can be modeled with motional resistance $(R)$, inductance $(L)$, and capacitance $(C)$ in electrical domain (Figure 1b). On the basis of the electrical circuit, the motion equation can be transferred into:

$$
L \frac{d i_{\mathrm{m}}}{d t}+R i_{\mathrm{m}}+\frac{1}{C} \int i_{\mathrm{m}} d t=V
$$

where $i_{\mathrm{m}}$ is the motional current, which presents the movement of the resonator, and $V$ is the applied force of resonator. Therefore, the resonant frequency can be calculated by the 
classical $L C$ resonant frequency Equation (7). The same result that a single resonator will be affected by the environmental influences can also be deduced in light of Equation (5).

$$
f=\frac{1}{2 \pi} \sqrt{\frac{1}{L C}}
$$

\subsection{Coupled Resonators}

\subsubsection{Dual Resonators Coupling}

The mass-stiffness-damper model of two coupled resonators is shown in Figure 2. The coupling strength is denoted by the coupling ratio $\left(\kappa=K_{\mathrm{c}} / K_{\text {eff }}\right)$. It was discussed in [55] that the system is recognized as strong coupling when $\kappa>1$ and weak coupling when $\kappa<0.1$. However, when $0.1<\kappa<1$, the weakly coupled and strongly coupled systems are not exactly defined. For weakly coupled resonators, frequencies of those two resonators are close, thus mode localization can form [126]. The output of the weakly coupled resonators is the eigenstates shift of those two resonators. However, due to the high spring constant of the coupling element, there is a difference for strongly coupled resonators, where the output of strongly coupled resonators is a frequency shift.

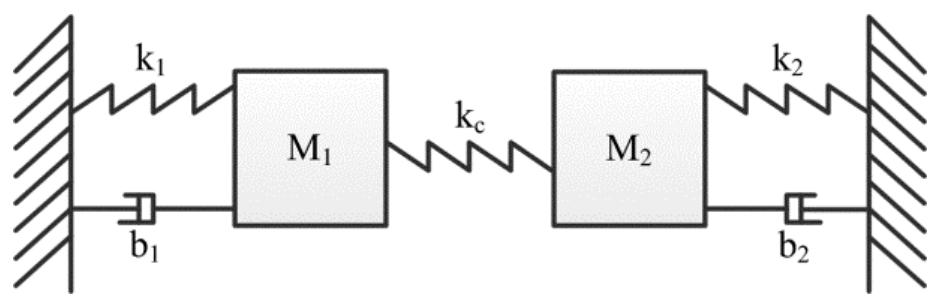

Figure 2. The mass-stiffness-damper model of coupled resonators.

The frequency shift of strongly coupled resonators has been deduced in reference [125]. The mass-stiffness-damper model of coupled resonators can be described by the equivalent electrical circuit (Figure 3a), where the coupling part can be divided into mechanical force coupling, electrostatic coupling, and magnetic coupling according to the different coupling strength. It can be further simplified by substituting the coupler part with the T-shape capacitor network (Figure 3b).

(a)

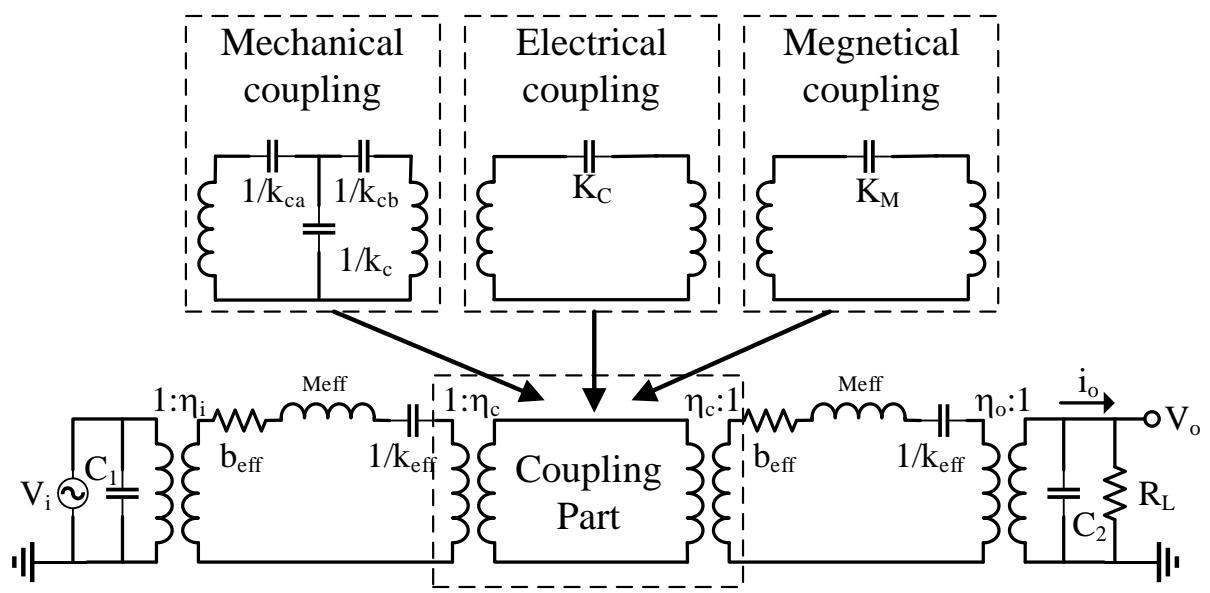

(b)

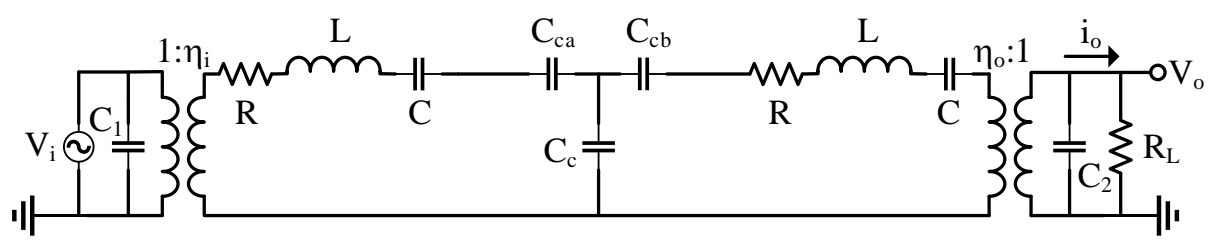

Figure 3. (a) Equivalent electrical circuit; (b) a simplified version of the circuit. 
The whole circuit can be expressed with a 2-rank matrix:

$$
\left[\begin{array}{cc}
\left(\left(s L_{x}+\frac{1}{s C_{x}}+R_{x}\right)+\frac{1}{s C_{\mathrm{ca}}}+\frac{1}{s C_{\mathrm{cc}}}\right) & -\frac{1}{s C_{\mathrm{cc}}} \\
-\frac{1}{s C_{\mathrm{cc}}} & \left(\left(s L_{x}+\frac{1}{s C_{x}}+R_{x}\right)+\frac{1}{s C_{\mathrm{ca}}}+\frac{1}{s C_{\mathrm{cc}}}\right)
\end{array}\right]\left[\begin{array}{l}
i_{1} \\
i_{2}
\end{array}\right]=\left[\begin{array}{c}
V_{i n} \\
V_{1}
\end{array}\right]
$$

The output of current over applied voltage can be obtained by solving this matrix.

$$
\begin{gathered}
\frac{i_{1}}{V_{1}}=\frac{\sigma+\frac{1}{s C_{\mathrm{ca}}}+\frac{1}{s C_{\mathrm{cc}}}}{\left(\sigma+\frac{1}{s C_{\mathrm{ca}}}\right)\left[\sigma+\frac{1}{s C_{\mathrm{ca}}}+\frac{2}{s C_{\mathrm{cc}}}\right]} \text {, where } \sigma=s L_{x}+\frac{1}{s C_{x}}+R_{x} \\
\frac{i_{2}}{V_{1}}=\frac{\frac{1}{s C_{\mathrm{cc}}}}{\left(\sigma+\frac{1}{s C_{\mathrm{ca}}}\right)\left[\sigma+\frac{1}{s C_{\mathrm{ca}}}+\frac{2}{s C_{\mathrm{cc}}}\right]}
\end{gathered}
$$

where $C_{\mathrm{ca}}, C_{\mathrm{cb}}$, and $C_{\mathrm{cc}}$ are the equivalent capacitors of coupling part, respectively. According to Equations (9) and (10), the frequency shift $(\Delta \mathrm{f})$ can be easily calculated.

The calculation of eigenstates changes in weakly coupled resonators have been clearly deduced in reference [123]. The normalized sensitivity of eigenstates changes can be expressed as:

$$
\begin{gathered}
\left|S_{M}\right|=\left|\frac{\partial \hat{u}}{\partial M} \times \frac{M}{\hat{u}}\right| \approx-\frac{K}{4 \kappa} \\
\left|S_{K}\right|=\left|\frac{\partial \hat{u}}{\partial K} \times \frac{K}{\hat{u}}\right| \approx-\frac{K}{4 \kappa}
\end{gathered}
$$

where, $u, M$, and $K$ are the eigenvector, mass, and stiffness, respectively. We can easily observe that the sensitivity is improved by $K /(2 \kappa)$ times than that of single resonator on the basis of Equations (3) and (4) [123]. Results also revealed that the sensitivity of eigenstates changes will be two to three orders higher than that of frequency variations when the coupling strength is two or three orders smaller than the stiffness $[56,74,123]$.

\subsubsection{Multiple Resonators Coupling}

The mass-stiffness-damper model of three coupled resonators is shown in Figure 4. The output calculation of three coupled resonators is different from that of two coupled resonators, of which the frequencies can be calculated with [55]:

$$
f_{1}=\frac{1}{2 \pi} \sqrt{\frac{k_{\text {eff }}}{M_{\text {eff }}}} ; f_{2}=f_{1} \sqrt{1+\kappa} ; f_{3}=f_{1} \sqrt{1+3 \kappa}
$$

where $K_{\text {eff }}=K_{1}=K_{2}=K_{3} ; M_{\text {eff }}=M_{1}=M_{2}=M_{3}$. And $f_{1}, f_{2}$, and $f_{3}$ are the frequencies of three resonators, respectively. According to Equation (13), the frequency shifts of strongly coupled resonators array can be easily calculated.

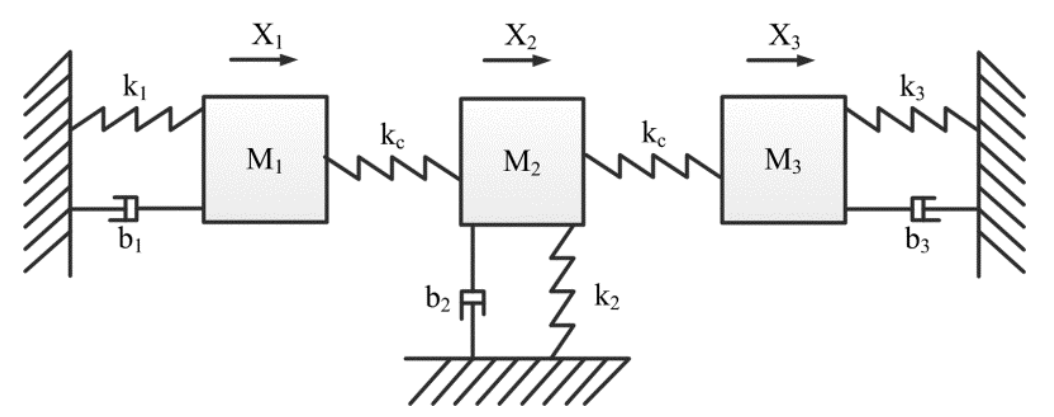

Figure 4. The mass-stiffness-damper model of three coupled resonators. 
For weakly coupled resonators array, the sensitivity of amplitude ratio can be denoted by [123]:

$$
\left|S_{K}\right|=|\partial| \frac{X_{1}}{X_{3}}|/ \partial| \frac{\Delta K_{\text {eff }}}{K_{\text {eff }}} \|=-\frac{K_{\text {eff }}\left(K_{2}-K_{\text {eff }}+K_{\mathrm{c}}\right)}{K_{\mathrm{c}}^{2}} \text { When } K_{\mathrm{c}}<\frac{K_{\text {eff }}}{10} \text { and } K_{2}>2 K_{\text {eff }}
$$

where, $K_{2}$, and $K_{\mathrm{c}}$ are the stiffness of resonator 2 and the coupling resonator, respectively. $X_{1}$ and $X_{3}$ are the movement of resonator 1 and 3, respectively. By comparing Equation (14) and Equation (12), we know that the normalized sensitivity of amplitude ratio of 3 weakly coupled resonators is improved by $4\left(K_{2}-K_{\text {eff }}+K_{\mathrm{c}}\right) / K_{\mathrm{c}}$, which is at least 40 times higher than the normalized sensitivity of eigenstates changes of 2 weakly coupled resonators [123]. Other works $[60,61]$ have also revealed that increasing the number of weakly coupled resonators is beneficial in improving its sensitivity. In order to be more intuitive and convincing, the comparison of single resonator, dual resonators and triple resonators are listed in Table 1.

Table 1. Properties of single resonator, dual and triple resonators.

\begin{tabular}{cccc}
\hline & Sensitivity of Frequency & Sensitivity of Eigenstate & \multicolumn{2}{c}{ Common Change Rejection } \\
\hline \multirow{2}{*}{ Single resonator } & $1 / 2$ & Non & Non \\
\hline \multirow{2}{*}{ Dual resonators } & Strongly-coupled & Weakly-coupled & \multirow{2}{*}{ Can } \\
\cline { 2 - 4 } & $K /(2 \kappa) /($ two to three orders) $[123]$ & $K /(2 \kappa)[123]$ & \\
\cline { 2 - 3 } Triple resonators & Strongly-coupled & Weakly-coupled & \multirow{2}{*}{ Can } \\
\cline { 2 - 4 } & Non & $4\left(K_{2}-K_{\text {eff }}+K_{\mathrm{c}}\right) / K_{\mathrm{c}}$ \\
\end{tabular}

\section{DRB and MRB MEMS Sensors}

DRB and MRB MEMS sensors can be realized by coupling resonators with strength, acoustic wave or by controlling resonators with feasible algorithm or hardware. On the basis of coupling methods, they can be categorized into three classes such as SCRB sensor, WCRB sensor and UCRB sensor. The following sections will analyze these three categories in detail.

\subsection{SCRB Sensors}

The first kind of DRB and MRB MEMS sensors are the SCRB sensors, whose resonators are mutually coupled by coupling strength. In fact, the coupling strength can be exerted by mechanical structure, electrostatic force, and magnetic force. According to these coupling methods, SCRB sensors can be further classified into three types: mechanical-strengthcoupled-resonator-based (ME-SCRB) sensors, electrical-strength-coupled-resonator-based (EL-SCRB) sensors, and magnetic-strength-coupled-resonator-based (MA-SCRB) sensors.

\subsubsection{ME-SCRB Sensors}

The resonators of the ME-SCRB sensor are directly connected by a mechanical element, as can be seen in Figure $5 a$. The beam with dimensions of width $W_{c}$, height $H_{c}$, and length $L_{\mathcal{C}}$ (Figure $5 b$ ) can be denoted by the T-shaped capacitor network in electrical domain $[127,128]$ (Figure 5c).

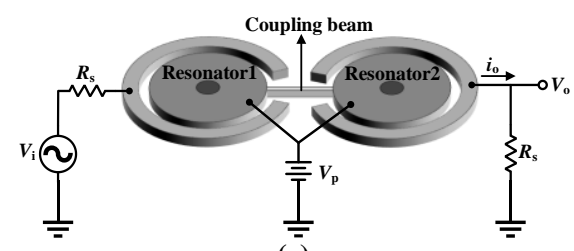

(a)

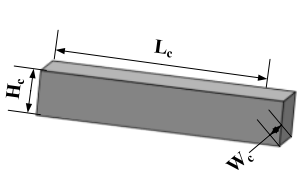

(b)

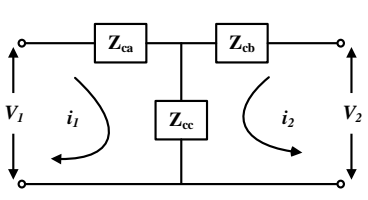

(c)

Figure 5. (a) Figure of mechanically coupled resonators; (b) Dimension of the coupling beam; and (c) T-shaped capacitor model for the coupling beam. 
The impedance value of each capacitor can be calculated by [125]:

$$
\begin{gathered}
Z_{c a}=Z_{c b}=\frac{\cos \left(2 \pi \cdot L_{c} / \lambda_{c}\right)-1}{j Z_{0} \sin \left(2 \pi \cdot L_{c} / \lambda_{c}\right)} \text { where } Z_{0}=\frac{1}{H_{c} W_{c} \sqrt{E \rho}} \\
Z_{c c}=\frac{1}{j Z_{0} \sin \left(2 \pi \cdot \lambda_{n}\right)} \text { where } \lambda_{n}=\frac{v_{e}}{f_{0}}=\frac{\sqrt{E / \rho}}{f}
\end{gathered}
$$

where $f_{0}$ is the resonant frequency, $Z_{0}$ and $\lambda_{n}$ are the normalized impedance and wavelength, respectively. $E$ and $\rho$ are the Young's modulus and density of the resonator material, respectively. On the basis of Equations (15) and (16), the coupling capacitances of Equations (9) and (10) can be determined when the beam works at the extensional mode [129]. The resonant frequency is different from $f_{0}$ through the addition or subtraction of $\mathrm{C}_{c a}$ to or from the motional capacitor of the constitute resonator, $C_{r}$. Then, the frequency shift with respect to the center resonant frequency $f_{0}$ can be calculated by:

$$
\frac{\Delta f}{f_{0}}=\sqrt{1+\frac{C_{r}}{C_{c a}}}-1 \cong \frac{C_{r}}{2 C_{c a}}
$$

It is obvious that the frequency shift between those two resonators can be controlled by changing the dimension of the coupling element.

Besides beam coupled resonators, other mechanical coupled resonators, such as corners coupled square resonators [130], middle side coupled Lame mode resonators, overhang coupling of beams and both-end connected same anchors [131], can also be used in ME-SCRB sensor $[55,132]$ as shown in Figure 6. On the basis of these structures, resonators are either weakly or strongly coupled to form a ME-SCRB sensor.

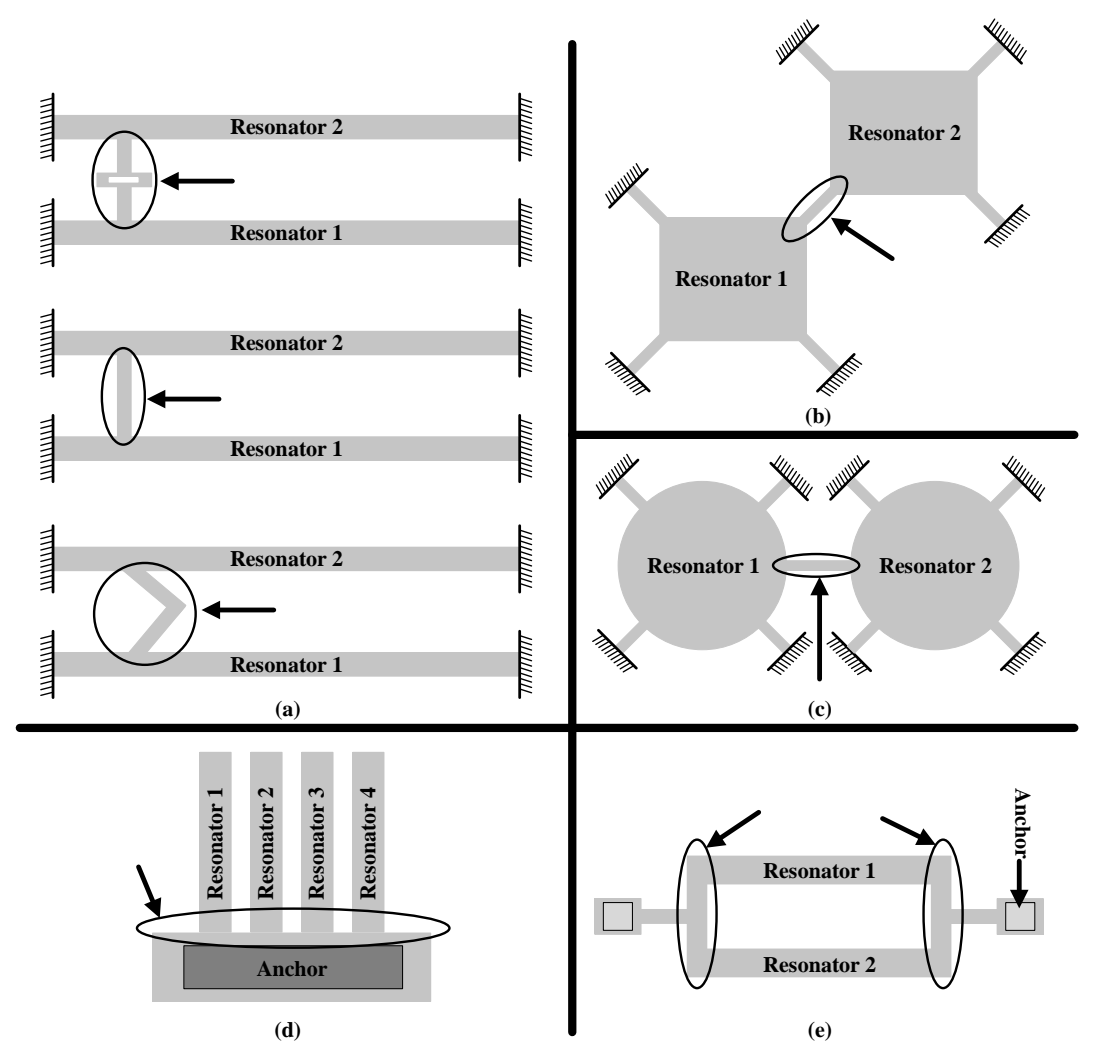

Figure 6. Structures of mechanical coupling of resonators. (a) Beam coupled; (b) Corner coupled; (c) Middle side coupled; (d) Overhang coupled; and (e) Double-end coupled. Reproduced with permission from [55]. Copyright (C 2014 by M. Sadegh Hajhashemi. 
Taking the coupling beam (Figure 6a) for example, we can define the coupling ratio ( $\kappa=K_{\mathrm{c}} / K_{\text {eff }}$ ) by the location relative to the vibration displacement. The coupling beam can be denoted by a matrix [55]:

$$
\begin{gathered}
{\left[\begin{array}{cc}
\frac{B \cdot c+C \cdot b}{B+b} & -\frac{2 E I_{c} \alpha^{3}(B \cdot c)}{j w L_{C}^{3}(B+b)} \\
-\frac{j w L_{C}^{3}(C \cdot c-1)}{E I_{c} \alpha^{3}(B+b)} & \frac{B \cdot c+C \cdot b}{B+b}
\end{array}\right]\left[\begin{array}{l}
F_{2} \\
\dot{x}_{2}
\end{array}\right]=\left[\begin{array}{l}
F_{1} \\
\dot{x}_{1}
\end{array}\right]} \\
b=\sin (\alpha X), c=\cos (\alpha X), B=\sinh (\alpha X) \text { and } C=\cosh (\alpha X) \\
X=\frac{x}{L_{c}} \text { and } \alpha^{4}=\frac{\omega^{2} L_{c}^{4} \rho A_{c}}{E I_{c}}
\end{gathered}
$$

where $\omega$ is frequency, $x$ is the vibration displacement, $A_{c}, L_{c}$, and $I_{c}$ are the cross section, length, and geometric moment of inertia of the coupling beam, respectively. $X$ is the normalized distance from the center line. It can be deduced that the coupling ratio will become weaker when the coupling beam moves from the center toward the anchor rod, as shown in Figure 7.

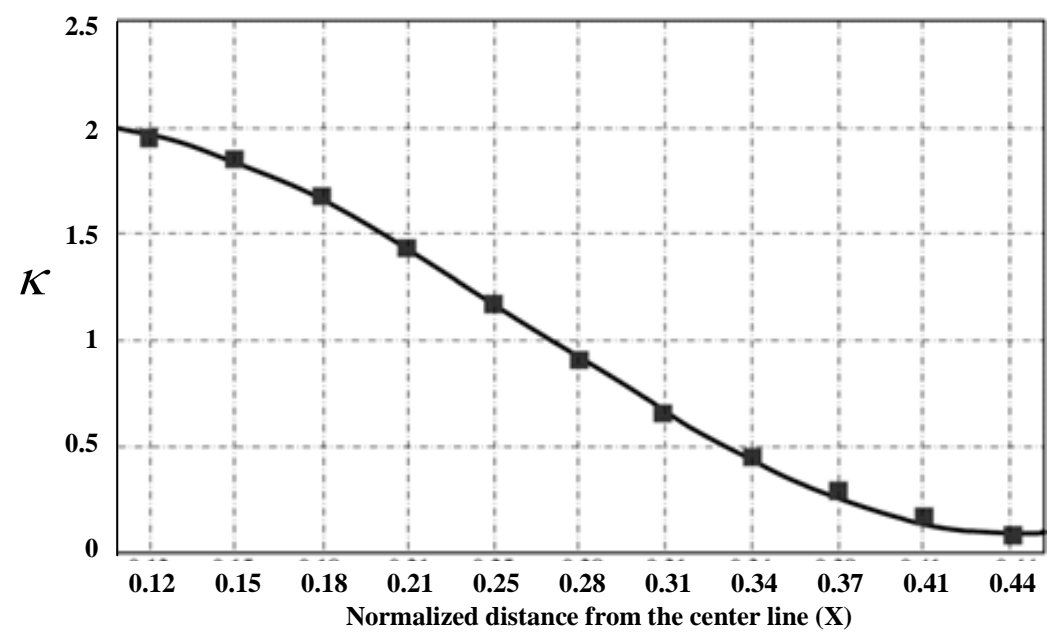

Figure 7. Coupling ratio of resonator varied with the location of beam. Reproduced with permission from [55]. Copyright (C) 2014 by M. Sadegh Hajhashemi.

During the last decade, a large number of ME-SCRB sensors have been developed such as mass sensors [55-58,68,69], electrometers [62,63] and acceleration measurement $[15,16,64-66,70]$.

In 2006, Spletzer et al. [56] developed the first weakly coupled ME-SCRB sensor for mass detection (Figure 8). It can produce a significant change in up to $5-7 \%$ in the eigenstates while the relative change in the resonant frequency is only $0.01 \%$, which illustrated that the sensitivity of eigenstate changes is 2 orders of magnitude higher than that of the frequency shift under ambient conditions. Ten years later, in 2016, Mohamad S. H. et al. [58] developed a strongly coupled ME-SCRB sensor for mass sensing (Figure 9). It is reported that the sensitivity of frequency shift of this sensor exceeds that of SRB sensor. Especially, the improvement in sensitivity is more than $20 \%$ when the coupling ratio equals 1.6. With the development of ME-SCRB sensors, in 2016, Honglong Chang et al. [62,63] developed a weakly coupled ME-SCRB sensor for electrical potential measurement (Figure 10a). Results showed that the sensitivity of eigenstate changes was improved by three orders of magnitude higher than that of frequency changes. Two years later, in 2018, Honglong Chang et al. [64] successfully developed a weakly coupled ME-SCRB sensor to measure acceleration for the first time (Figure 10b). The sensor exhibited good properties by using amplitude ratio shift as output. Results showed that the sensitivity of amplitude ratio $(\sim 312,162 \mathrm{ppm} / \mathrm{g})$ is approximately 302 times higher than that of the frequency change ( 1035 ppm/g). 

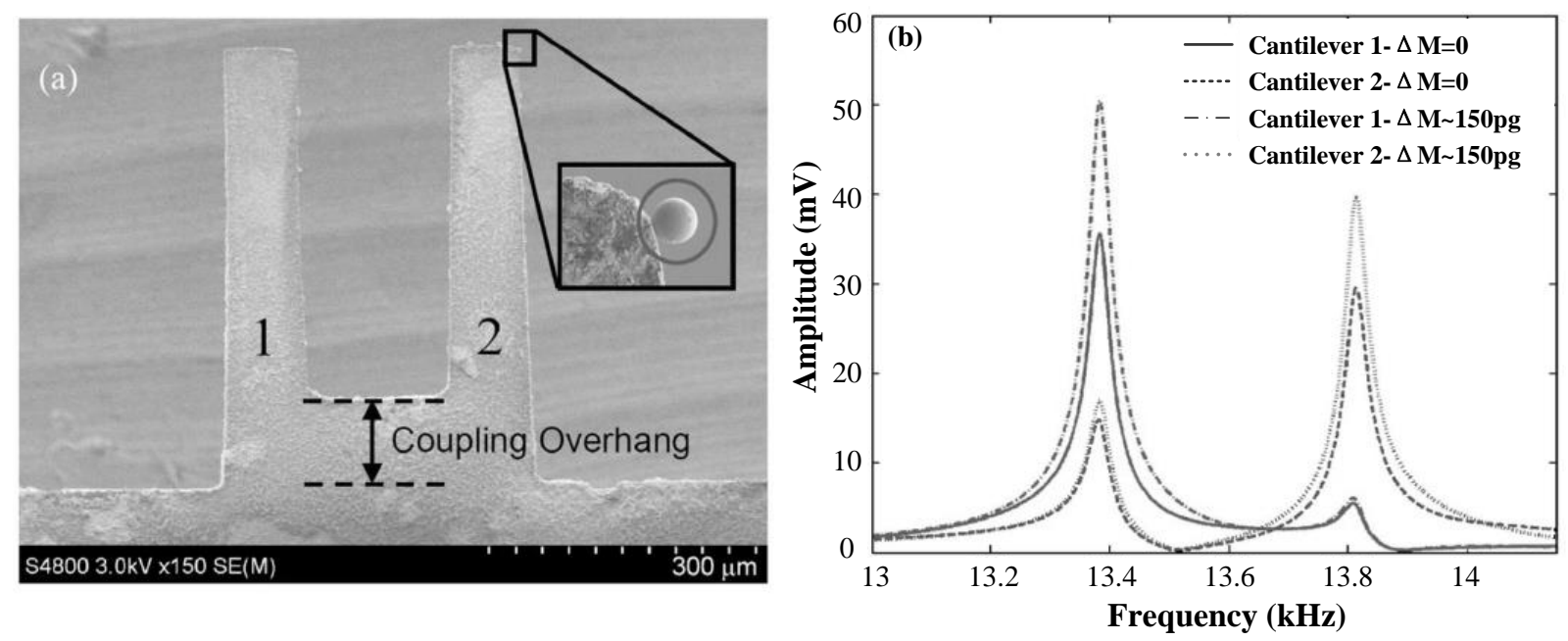

Figure 8. (a) SEM image of the coupled cantilevers resonator; (b) frequency change in coupled resonator before and after the induced mass. Reproduced with permission from [56]. Rights managed by AIP Publishing.

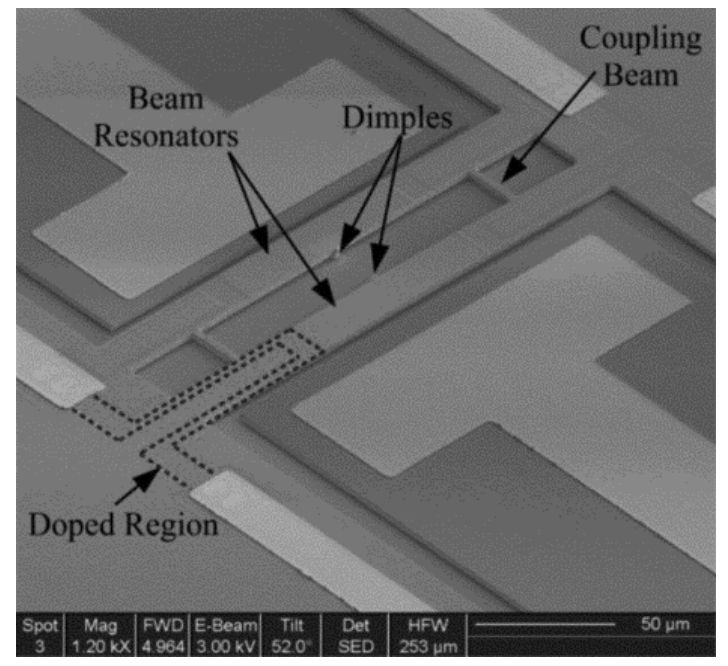

Figure 9. SEM image of the coupled cantilevers resonator. Reproduced with permission from [58]. Copyright () 2016, IEEE.
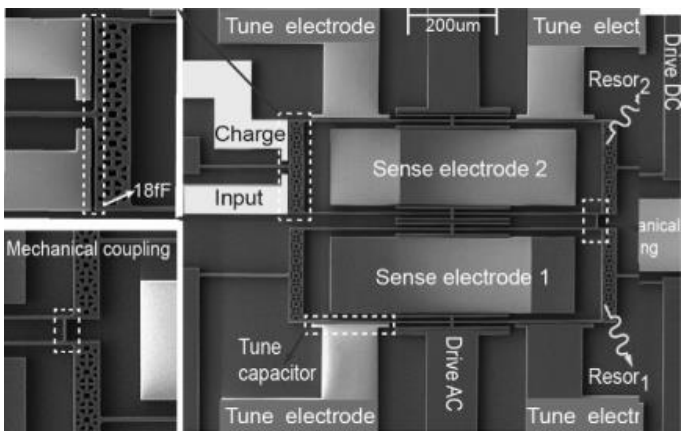

(a)

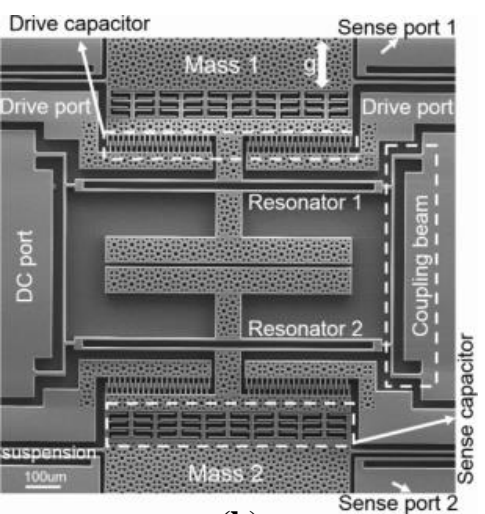

(b)

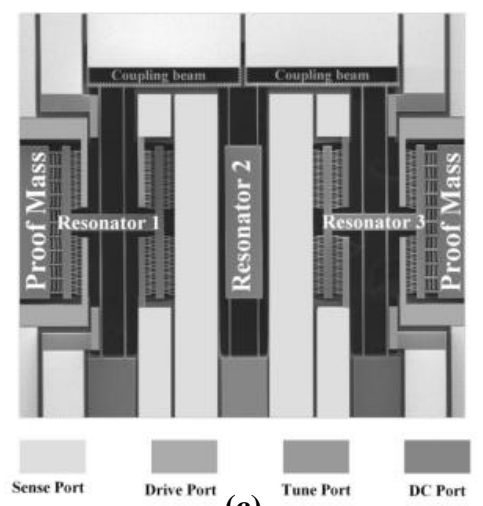

(c)

Figure 10. SEM image of two resonators weakly coupled ME-SCRB sensor: (a) electrometer; Reproduced with permission from [63]. Copyright (C) 2016, IEEE. (b) accelerometer; Reproduced with permission from [64]. Copyright (C) 2015, IEEE. (c) three resonators weakly coupled ME-SCRB sensor for acceleration measurement. Reproduced with permission from [66]. Copyright (c) 2018, IEEE. 
It can be concluded from the above-mentioned research works that ME-SCRB sensors with dual resonators have exhibited good performance. Besides, ME-SCRB sensors with three resonators have also been developed. In 2018, Honglong Chang et al. [66] successfully developed a weakly coupled ME-SCRB sensor with three resonators to measure acceleration for the first time (Figure 10c). In this sensor, resonators are weakly coupled by two coupling beams. When inertial perturbation acts on the proof mass, the sensor will produce shifts in amplitude ratio due to the mode localization between the two outer resonators. It is reported that the sensitivity of amplitude ratio of this accelerometer is improved by $348 \%$ in comparison with that of eigenstate changes of the accelerometer. Other ME-SCRB sensors with no less than three resonators have also developed. In 2008, Spletzer et al. [57] fabricated a fifteen-cantilever array as a weakly coupled ME-SCRB sensor (Figure 11a). It is reported that the magnitude of eigenvector has a large relative change in the order of $10-100 \%$ due to the added mass, while the highest relative frequency shift is only about $0.1 \%$. The relative shift of eigenvector was two to three orders higher than the relative frequency shift. Six years later, in 2014, Mohamad S. H. et al. [55] reported a cantileverarray-based strongly coupled ME-SCRB sensor for mass measurement (Figure 11b). In the second mode of vibration, a significant frequency shift can be observed in this sensor, which also proved that the sensitivity of cantilever-array-based strongly coupled ME-SCRB sensor exceeds that of a SRB sensor.

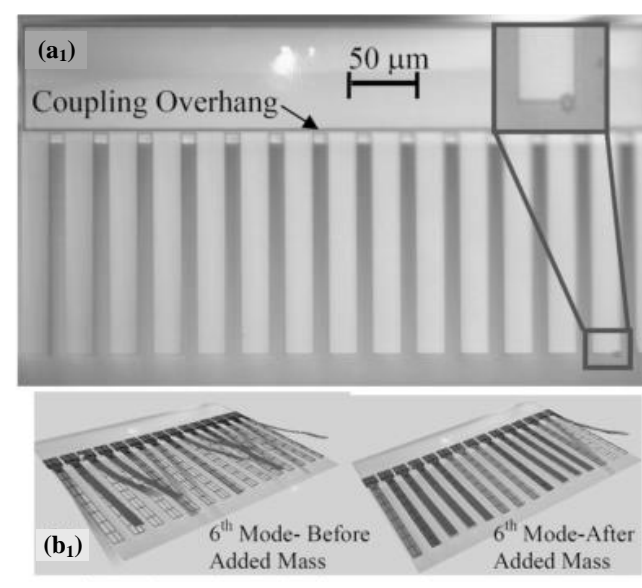

(a)

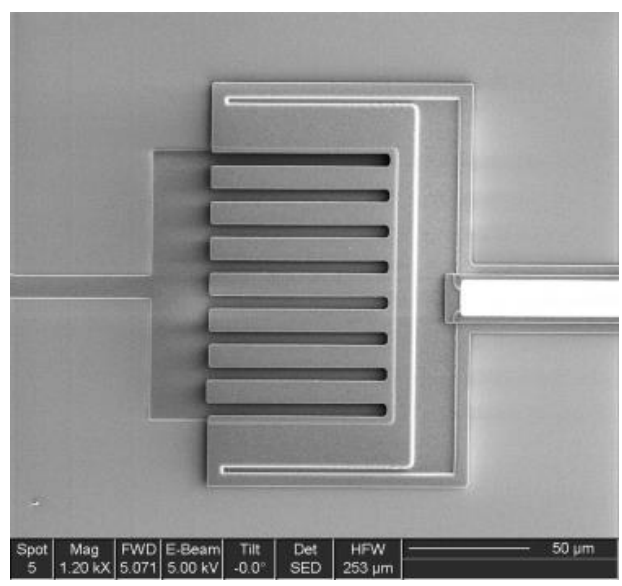

(b)

Figure 11. SEM photo of (a) weakly coupled ME-SCRB with cantilever array. Reproduced with permission from [57]. Rights managed by AIP Publishing. (b) Strongly coupled ME-SCRB with cantilever array. Reproduced with permission from [55]. Copyright ( 2014 by M.Sadegh Hajhashemi.

The comparisons of the properties of different ME-SCRB sensors are listed in Table 2. We can draw the conclusion that the strong or weak coupling has enabled the ME-SCRB sensors a good performance. According to the abovementioned works, we can also conclude that one of the biggest advantages of ME-SCRB sensors is that, once resonators are connected, no further action is needed to couple them together. Although the tuning of the coupling strength is difficult, the ME-SCRB sensors are promising in the field of high-end sensors. However, deviations may occur in the device due to the fabrication errors. Hence, researchers are still sparing no effort to optimize these ME-SCRB sensors 
Table 2. Comparisons of properties of different ME-SCRB sensors.

\begin{tabular}{|c|c|c|c|c|c|}
\hline \multirow{2}{*}{$\begin{array}{c}\text { Number of Coupled } \\
\text { Resonators }\end{array}$} & \multirow{2}{*}{ Sensor } & \multirow{2}{*}{$\begin{array}{l}\text { Change of } \\
\text { Frequency }\end{array}$} & \multirow{2}{*}{$\begin{array}{c}\text { Weakly Coupled } \\
\text { Shift of Eigenstates }\end{array}$} & \multirow{2}{*}{$\begin{array}{c}\text { Strongly Coupled } \\
\text { Beat Frequency }\end{array}$} & \multirow{2}{*}{ Improvement } \\
\hline & & & & & \\
\hline \multirow{6}{*}{$\begin{array}{l}\text { Dual } \\
\text { resonators }\end{array}$} & Mass sensor [56] & $0.01 \%$ & $5-7 \%$ & - & two orders \\
\hline & Mass sensor [58] & - & - & yes & $20 \%$ \\
\hline & Electrometers [63] & $283.56 \mathrm{ppm}$ & $663,751 \mathrm{ppm}$ & - & three orders \\
\hline & Accelerometer [64] & $1035 \mathrm{ppm} / \mathrm{g}$ & $312,162 \mathrm{ppm} / \mathrm{g}$ & 一 & 302 times \\
\hline & Mass sensor [68] & $0.03 \% / \mathrm{pg}$ & $2.5 \% / \mathrm{pg}$ & - & two orders \\
\hline & Mass sensor [69] & -0.17 & 422 & - & 2482 times \\
\hline \multirow{3}{*}{$\begin{array}{l}\text { Multiple } \\
\text { resonators }\end{array}$} & Mass sensor [57] & $0.1 \%$ & $10-100 \%$ & - & two to three orders \\
\hline & Mass sensor [55] & $4.2 \mathrm{kHz}$ & 一- & $\begin{array}{l}\text { High coupling ratio } \\
\text { High sensitivity }\end{array}$ & - \\
\hline & Accelerometer [66] & $11.46 \mathrm{~Hz} / \mathrm{g}$ & $705,000 \mathrm{ppm} / \mathrm{g}$ & - & 1410 times \\
\hline
\end{tabular}

\subsubsection{EL-SCRB Sensors}

Resonators of EL-SCRB sensors are mutually coupled by electrostatic force. Similarly to the coupling element of mechanically coupled resonators, the coupling capacitor of electrically coupled resonators can also be substituted with the T-shape capacitor network in the lumped element model. Hence, the frequency change can be calculated following the Equations (8)-(10). However, the frequency shift is not good enough to describe the properties of weakly coupled resonators. In 2016, Chun Zhao [123] summarized the deduction processes of the shift of eigenstates for two and three coupled resonators and that of the shift of amplitude ratio for three coupled resonators. Basically, all of the the ELSCRB sensors are developed on the basis of these theoretical results. Electrically coupled resonators were first introduced in MEMS filter by Pourkamali and Ayazi [76] in 2005. Four years later, in 2009, the first EL-SCRB sensor was designed by the group of A. Seshia [74].

From 2010 to 2012, the group of A. Seshia have developed several EL-SCRB sensors including mass sensor [77], displacement sensor [78] and electrometer [79] as shown in Figure 12a-c. Results illustrated that relative shift of eigenstates of EL-SCRB sensor for mass sensing [77] is about $4.32 \%$ and $3.448 \%$ at the two eigenvalues, respectively, while the relative variation of frequency is only about $0.00237 \%$, which proved the much higher sensitivity of eigenstates shift than that of the frequency shift. Such conclusion has also been proved in EL-SCRB sensors for displacement measurement [78] and charge sensing [79] application. The relative shifts of eigenstates are two or three orders of magnitude higher than that of frequency shift. Recently, M. Lyu [80] proposed a novelty mass sensor with distributed electrodes based on the mode localization of two electrostatically coupled microbeams in higher-order modes. Compared to the output metric of frequency shift, the sensitivity improved by four orders of magnitude by using the amplitude ratio as the output.

The comparisons of properties of different EL-SCRB sensors are listed in Table 3. In addition to the high sensitivity property, these EL-SCRB sensors can also obtain the capability of common mode rejection, because the eigenstates of those coupled resonators are affected to the same extent. 


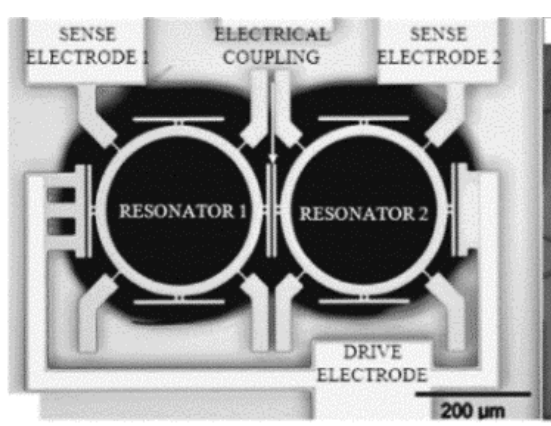

(a)

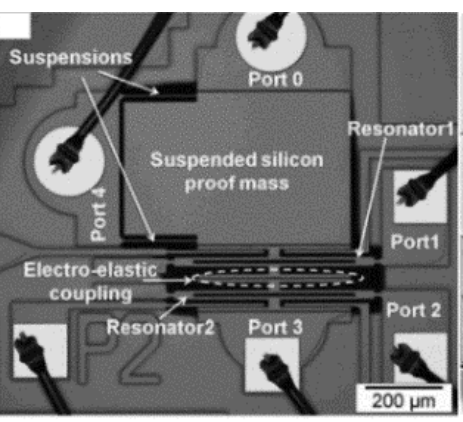

(b)

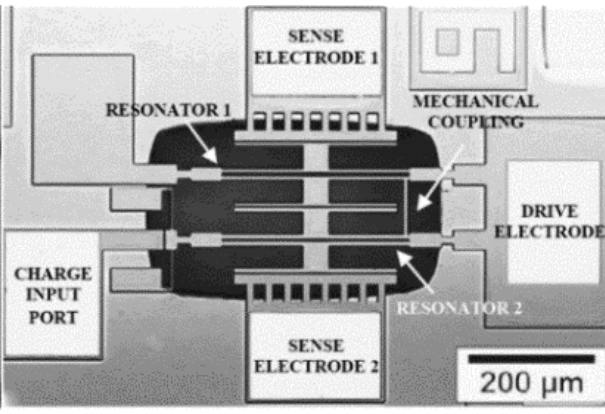

(c)

Figure 12. The Photos of electrically coupled resonators (a) mass sensor. Reproduced with permission from [77]. Rights managed by AIP Publishing. (b) displacement sensor. Reproduced with permission from [78]. Copyright (C 2012, IEEE. (c) electrometer. Reproduced with permission from [79]. Copyright @ 2010, IEEE.

Table 3. Comparisons of properties of different EL-SCRB sensors with two resonators.

\begin{tabular}{cccc}
\hline Sensor & Shift of Frequency & Shift of Eigenstates & Improvement \\
\hline Mass sensor [77] & $0.00237 \%$ & $4.32 \%$ or $3.448 \%$ & Two orders \\
\hline Displacement sensor [78] & maximum $0.005 \%$ & maximum $1.8 \%$ & Three orders \\
\hline Electrometer [79] & maximum $0.006 \%$ & maximum $1.8 \%$ & Nearly three orders \\
\hline Mass sensor [80] & $0.02 \%$ & $221 \%$ & More than four orders \\
\hline
\end{tabular}

The EL-SCRB sensors with three resonators are also studied. From 2015 to 2018, Chun Zhao et al. have developed several EL-SCRB sensors with three resonators (Figure 13) for mass [81], stiffness [84-86] and force [87] sensing applications. Results showed that when the EL-SCRB sensor is used for mass sensing application, the sensitivity of the change in the amplitude ratio is around two orders of magnitude larger than that of frequency shift and is about twice of amplitude change. When the EL-SCRB sensor is used as a stiffness sensor [84], the highest normalized sensitivity is 13,558, which has been improved at least 56 times than that of the EL-SCRB sensor with 2 resonators. The further application of EL-SCRB sensor in force detection [87] has also been studied. Results showed that the sensitivity is two orders of magnitude higher than that of the conventional single resonator force sensor. The comparisons of the EL-SCRB sensors for mass, stiffness, and force detections are listed in Table 4 . We can draw the conclusion from the data of Table 4 that the sensitivities of the EL-SCRB sensors have been much improved compared with those of single resonator sensors, and increasing the number of coupled resonators is beneficial to further improve its sensitivity, which is the great merit of multiple coupled resonators.

Table 4. Comparisons of EL-SCRB sensors for stiffness and force detections.

\begin{tabular}{|c|c|c|c|c|c|}
\hline \multirow{2}{*}{ Sensor Type } & \multirow{2}{*}{ Reference } & \multirow{2}{*}{ Output of Sensor } & \multirow{2}{*}{ Amplitude Ratio } & Improvement & Improvement \\
\hline & & & & As Frequency Shift & As Eigenstate Shift \\
\hline \multirow{3}{*}{ Mass sensor } & [81] & Amplitude ratio & $25.31 \%$ & Nearly two orders & Nearly two times \\
\hline & {$[82]$} & Amplitude ratio & $0.4 \%$ & Nearly two orders & - \\
\hline & [83] & Amplitude ratio & 35.6 & More than three orders & - \\
\hline \multirow{3}{*}{ Stiffness sensor } & [84] & Amplitude ratio & 13,558 & More than three orders & 56 times \\
\hline & [79] & Shift of eigenstates & 275 & Two orders & - \\
\hline & [133] & Shift of frequency & 0.5 & - & 一 \\
\hline \multirow{3}{*}{ Force sensor } & [87] & Amplitude ratio & $4.9 \mathrm{e} 6 / \mathrm{N}$ & Two orders & More than three orders \\
\hline & [79] & Shift of eigenstates & $1478 / \mathrm{N}$ & - & - \\
\hline & [134] & Shift of frequency & $8995 / \mathrm{N}$ & - & - \\
\hline
\end{tabular}




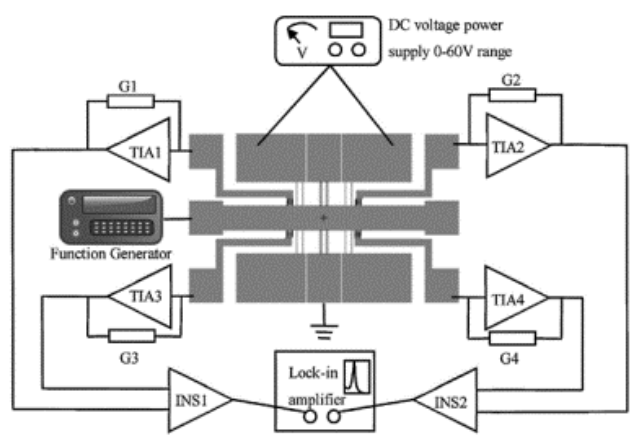

(a)

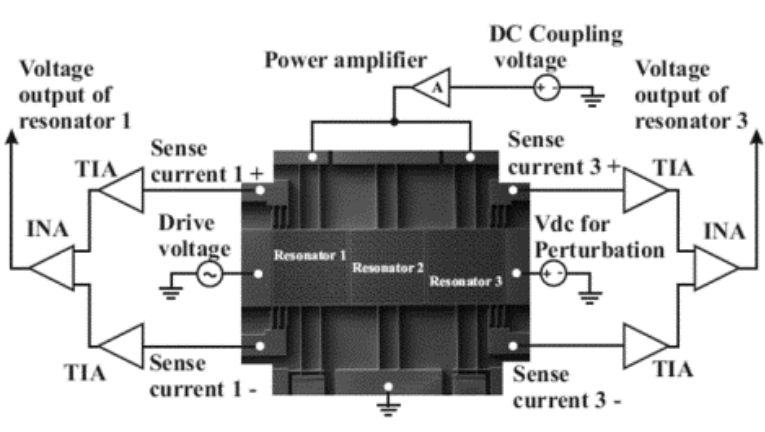

(b)

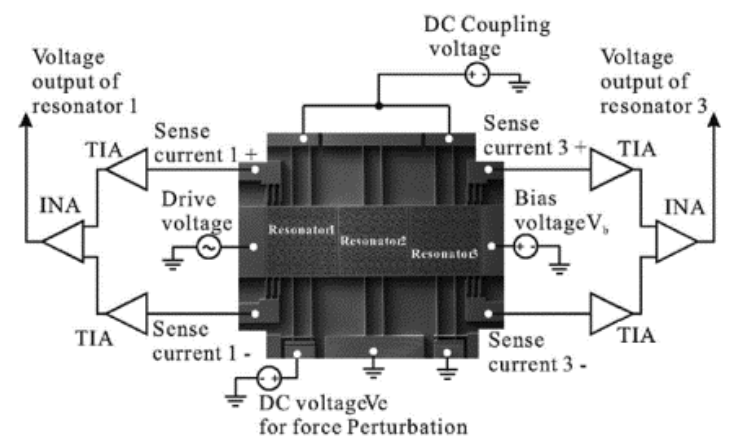

(c)

Figure 13. The electrically coupled three resonators (a) mass sensor. Reproduced with permission from [81]. (C) 2018 Elsevier B.V. (b) stiffness sensor. Reproduced with permission from [84]. Copyright @ 2015, IEEE. (c) force sensor. Reproduced with permission from [87]. (C) 2015 Elsevier B.V.

Through a comprehensive analysis of these studies of EL-SCRB sensors, we can observe that the biggest advantage of this kind of sensor is that the coupling strength can be easily tuned. However, when the difference between the resonant frequencies of the two resonators is too small, the two resonant modes will interfere with each other. This phenomenon, which is termed as mode-aliasing [87], should be avoided in the sensor design process. The anti-mode-aliasing condition has been found in reference [87]. Reference [87] also pointed out that the impractical for real time application, low Q-factor resonators and high noise are the three limitations of EL-SCRB sensors. Fortunately, Chun Zhao et al. have been working on resolving those above problems. In 2018, they proposed a noise optimization method [88] with an optimal region for the resolution existing in vicinity of amplitude ratio 1.22. However, a trade-off between resolution and linearity still exists as the optimal region is not the linearity part.

\subsubsection{MA-SCRB Sensors}

Resonators of MA-SCRB sensors are mutually coupled by magnetic force. Generally, the distance between electrically and mechanically coupled resonators is in the range of $1 \mathrm{~nm}$ to $1 \mu \mathrm{m}$. From the fabrication point of view, it is hard to uniformly manufacture them due to the uneven manufacturing processes. In order to resolve such problem, Pai et al. [91] developed a MA-SCRB sensor, which is used as gyroscope with large separations. In this sensor, neodymium permanent magnets, installed on the proof mass, are used to form a magnetic coupling, as shown in Figure 14b. The actuation and sensing of the resonators are realized through electrostatic transduction, as shown in Figure 14c. In summary, the MA-SCRB sensor is feasible. However, much work needs to be conducted before it can be put into practical use. 

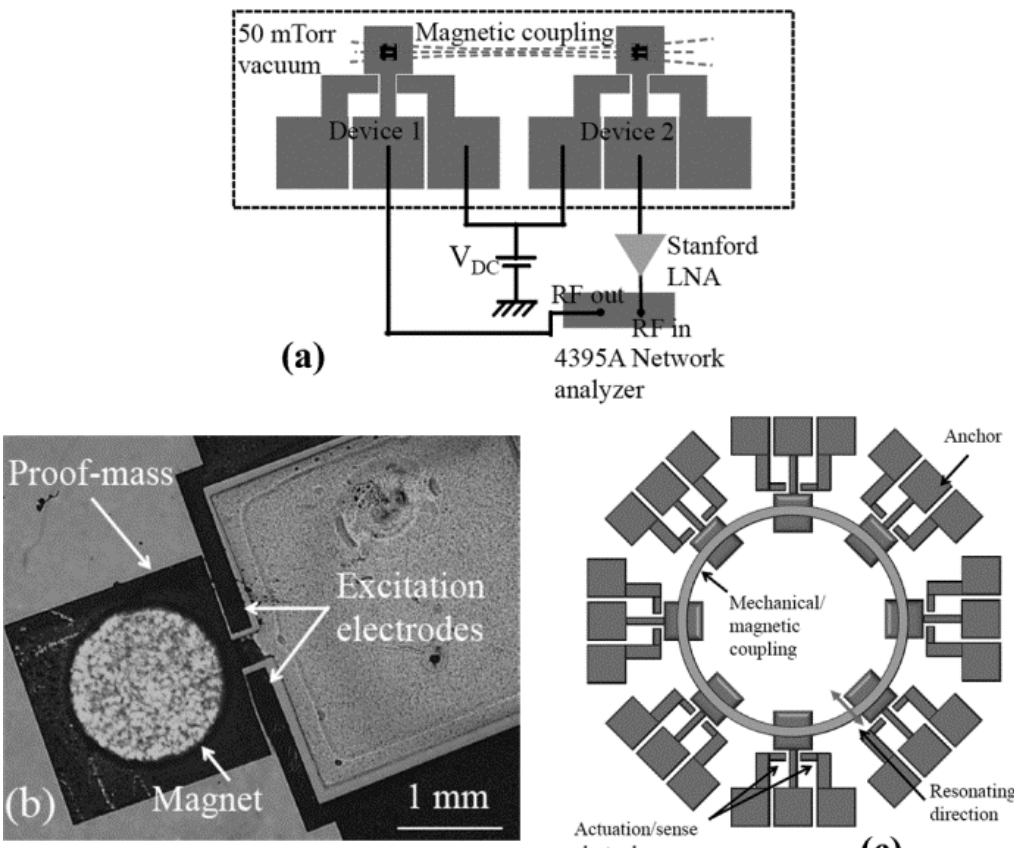

electrodes

(c)

Figure 14. The magnetically coupled resonators: (a) Schematic of the experimental setup; (b) Optical microscope image; (c) A conceptual gyroscope. Reproduced with permission from [89]. Copyright (C) 2014, IEEE.

\subsubsection{Common Mode Rejection of SCRB Sensors}

The ability of common change rejection of coupled resonators has been investigated. Reports showed that environmental factors (such as ambient pressure $[90,91]$ and/or temperature [92-95]) or nonspecific bindings (for mass sensing) influence all of the vibrating elements uniformly, ideally not affecting the eigenmodes of the system, while shifts in the resonance frequencies still occur. One of the early works addressing the common-mode rejection of mode-localized sensor is provided in [90]. Experimental results demonstrated its intrinsic common-mode-rejection ability. Other work in [91] also demonstrated the ambient pressure drift rejection capability of the mode-localized sensors. Results showed that amplitude ratio (AR) based output remained relatively insensitive against the ambident pressure drift compared with frequency shift output. The maximum error of AR based output in sensitivity was $2.74 \%$, whereas that of the frequency shift output reached to about $21.6 \%$ for a pressure range of 2.6 to $20 \mathrm{~Pa}$. In addition to ambient pressure, mode-localized sensors also have the ability to suppress the impact of environmental temperature on the sensors. Results in [92] presented that when temperature changes, maximum measurement error in the AR output is $8.8 \%$ whereas maximum error in measurement of frequency shift output is $>1000 \%$. Results in [93] also demonstrated the ability of mode-localized sensor in immunity to temperature fluctuations (between $35^{\circ} \mathrm{C}$ and $60^{\circ} \mathrm{C}$ ). Other works $[94,95]$ also revealed that the AR has an excellent temperature drift suppression capability compared to the frequency shift output. The reason for the proposed advantage of using AR shift output is that any ambient variable (for instance temperature/pressure) will equally affect the output sine waveform of each of the resonators. Therefore, environmental effect is cancelled to the first order with the ratio-based output.

\subsubsection{Resolution of SCRB Sensors}

Owing to the mode localization phenomenon of weakly coupled resonators (WCRs), the sensitivity of the amplitude ration (AR) output metric is at least two or three orders of magnitude higher than that of the resonant frequency, which has been theoretically and experimentally demonstrated by various types of WCRs-based resonant sensors. Nevertheless, to what extent the mode-localized sensing paradigm influences the ultimate 
resolution remains unknown. Additionally, this idea even was regarded as the most important fundamental research question in this field in 2018.

Zhang et al. reported a high-sensitivity resonant electrometer [63] based on the mode localization of two degree-of-freedom (DoF) WCRs. The experimental results showed that the sensitivity enhancement is more than three orders of magnitude when selecting the amplitude ratio instead of the resonant frequency as the output metric. However, the resolution of the electrometer using both frequency and amplitude ratio readouts is in the same range. They also reported an acceleration sensing method [65] based on two WCRs and demonstrated that the measured relative shift in amplitude ratio was 302 times higher than the shift in resonance frequency but the improvement in the resolution was not obvious. M. Pandit [73] explored coupled nonlinear MEMS resonators as sensors based on the principle of energy localization, an improvement of $2 \times$ in input-referred noise floor, or resolution, in comparison to its linear counterpart at both the bifurcation points, has been achieved around veering and an improvement of $4 \times$ at the top bifurcation point away from veering despite of reduced sensitivity. This is likely due to the higher Signal to Noise Ratio (SNR) and additional noise filtering properties at the bifurcation points.

Regarding the problem of resolution of mode-localized sensors, there are mainly two different views. J. Juillard et al. $[96,97]$ hold the view that mode-localized sensors based on the amplitude ratio output metric provide measurements whose resolution is independent on coupling strength, while A. Seshia [98] and Chang [99] believe that that the resolution of mode-localized sensors is dependent on the strength of internal coupling $k$. They believe that weaker effective coupling (lower $k$ ) between the resonators should help enhance not only the sensitivity of such sensors, but also contribute to substantial improvements in the resolution. Different views of resolution limit models are shown in Table 5.

Table 5. Comparison of resolution limit model of 2-DoF WCRs.

\begin{tabular}{cc}
\hline Reference & Resolution Limit Model \\
\hline J. Juillard [96,97] & $R_{\mathrm{AR}}=\frac{2 N}{Q F} \Delta f^{\frac{1}{2}}$ \\
\hline A. Seshia [98] & $R_{\mathrm{AR}} \approx 8 \kappa \sqrt{\frac{\frac{E}{\mathrm{th}}_{\mathrm{t}} \Delta f}{2 E_{\mathrm{c}} Q \omega_{\mathrm{r}}^{\mathrm{eff}}}}$ \\
\hline Chang [99] & $R_{\mathrm{AR}} \approx 2 \sqrt{2} \kappa \frac{N}{F} \Delta f^{\frac{1}{2}}=2 \sqrt{2} \kappa \frac{\sqrt{4 \kappa_{\mathrm{B}} T_{\mathrm{c}} \Delta f}}{F}$ \\
\hline
\end{tabular}

Chang [99] also pointed out that the resolution of AR-based mode-localized sensors can be improved by increasing DoFs in the resonant system, and the resolution limit of the high-order (the DoFs > 3) mode-localized sensors using the AR output metric is better than that of the frequency-output metric, as shown in Table 6. They revealed that the 3-DoF mode-localized sensors using the AR output metric have a better resolution limit than both the 2-DoF and frequency-output sensors, and the 4-DoF sensors indicate the best resolution limit, which is three orders better than 3-DoF. This trend coincides with the experimental data in [98,104,105], of which the resolution is improved from $8000 \mathrm{e} / \sqrt{ } \mathrm{Hz}(2 \mathrm{DoF})$ [100] to $9.21 \mathrm{e} / \sqrt{ } \mathrm{Hz}(3-\mathrm{DoF})[100]$ and $0.256 \mathrm{e} / \sqrt{ } \mathrm{Hz}(4-\mathrm{DoF})[101]$.

Table 6. Comparison of resolution limit model using frequency and AR output metrics. Adapted from [99].

\begin{tabular}{ccc}
\hline \multicolumn{2}{c}{ Output } & Resolution Limit Model \\
\hline \multicolumn{2}{c}{ Frequency Output Metric } & $\frac{1}{Q} \frac{N}{F} \Delta f^{\frac{1}{2}}$ \\
\hline & 2-DoF & $2 \sqrt{2} \kappa \frac{N}{F} \Delta f^{\frac{1}{2}}$ \\
\cline { 2 - 3 } AR Output Metric & 3-DoF & $\frac{2 \sqrt{2} \kappa^{2}}{a-1} \frac{N}{F} \Delta f^{\frac{1}{2}}$ \\
\cline { 2 - 3 } & 4-DoF & $\frac{2 \sqrt{2} \kappa^{3}}{(a-1)^{2}} \frac{N}{F} \Delta f^{\frac{1}{2}}$ \\
\hline
\end{tabular}


Overall, the resolution of mode-localized sensors is related to multiple factors such as sensitivity, noise, and quality factors; we can improve the resolution by further increasing the sensitivity and reducing the amplitude noises both of extrinsic noise arising from the external electronic interfacial readout circuitry, and intrinsic noises that are inherent to the micro- or nano-mechanical resonator arrays.

\subsubsection{Summary}

In this section, three different types SCRB sensors, named as ME-SCRB sensor, ELSCRB sensor and MA-SCRB sensor, were introduced in detail, respectively. In general, all of these SCRB sensors have the merit of high sensitivity and common change rejection, but the resolution improvement is not obvious, and there is still a lot of room for enhancement. Besides, there are still other more or less shortcomings that need to be resolved. The ME-SCRB sensor has the disadvantage of bad tunability of coupling strength; the EL-SCRB sensor has the weak points of low Q-factor resonators and high noise; the MA-SCRB sensor is too far away from real application. In addition, they are all limited by the fabrication processes. A good fabrication process can accurately control the coupling strength of the ME-SCRB sensor, improve the Q-factor of the EL-SCRB sensor, and reduce the nonuniformities of magnets fabrication in the MA-SCRB sensor. Therefore, it is necessary to spare no effort in the precision manufacturing process to make these sensors get practical applications in the future.

\subsection{WCRB Sensors}

The second kind of DRB and MRB MEMS sensors are the WCRB sensors, of which resonators are acoustically coupled. Generally, the resonators of WCRB sensor can be thought as acoustic waveguides [109-111]. The dimension-related properties of waveguides determine the types of propagated acoustic waves. It offers an opportunity for different resonant modes which can be coupled together with the same frequency. In 2013, Roozbeh Tabrizian et al. [109] proved that due to the dispersion characteristics of the Lamb wave, different resonant modes of a Lamb wave resonator (LWR) can be coupled into a whole sensor structure by engineering the width of resonator (Figure 15) [109]. Results in [109] showed that by extracting a small beat frequency from an integer combination of two resonance modes with large difference in their temperature coefficient of frequency (TCF), a high TCF of $\sim 8300 \mathrm{ppm} /{ }^{\circ} \mathrm{C}$ was achieved, which demonstrated the suitability of acoustically coupled resonators for temperature sensing with high accuracy and resolution.

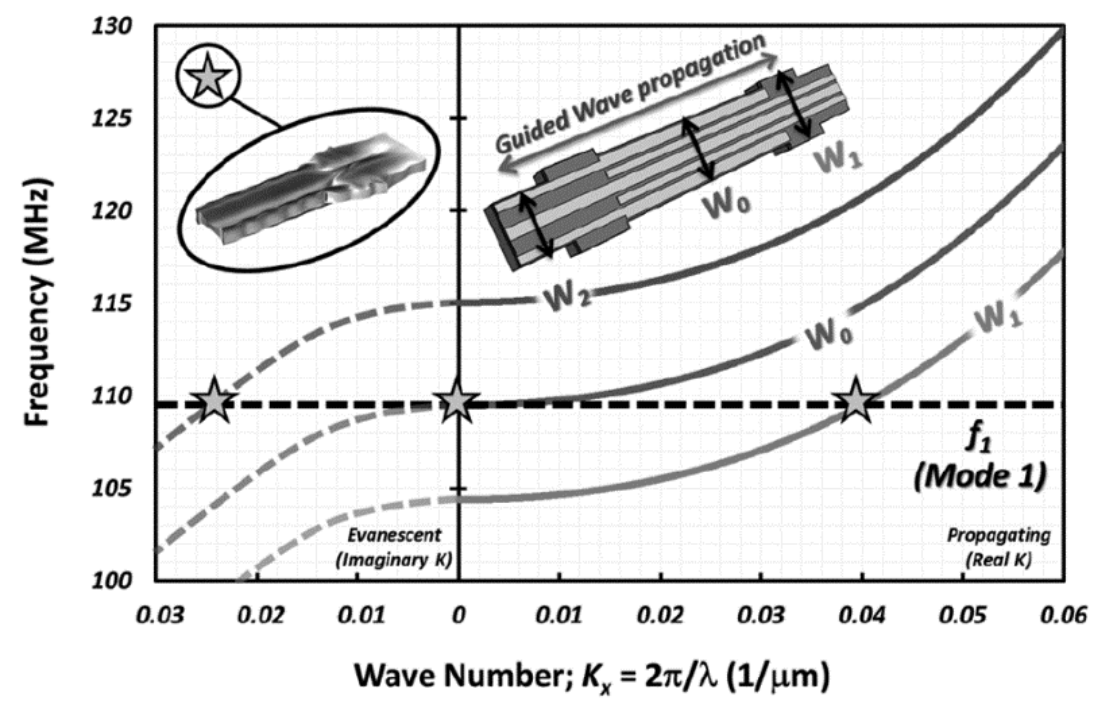

Figure 15. Dispersive behavior of propagating waves. Reproduced with permission from [109]. Copyright @ 2013, IEEE. 
For other specific LWR that vibrates in the coupling of first-order width extensional mode (WE1) and the second-order width shear mode (WS2), the width of each part, $\left(W_{1}\right.$, $W_{2}$ ) can be calculated based on the same frequency of different parts:

$$
\begin{gathered}
f_{1}=f_{2} \Rightarrow \frac{V_{2}}{W_{2} / 2}=\frac{V_{1}}{W_{1}} \Rightarrow \frac{W_{2}}{W_{1}}=\frac{2 V_{2}}{V_{1}} \text { where } V=\sqrt{\frac{C}{\rho}} \\
\Rightarrow \frac{W_{2}}{W_{1}}=\sqrt{\frac{4 C^{\prime}}{C^{\prime \prime}}}=\sqrt{\frac{4\left(C_{11}-C_{12}\right)}{C_{11}+C_{12}+2 C_{44}}}
\end{gathered}
$$

where $C^{\prime}$ is the quasi-shear elastic constant in shear modes and $C^{\prime \prime}$ is the quasi-longitudinal elastic constant in extensional modes.

Low temperature coefficient of frequency (TCF) is one of the merits of the WCRB sensor, which can be realized by coupling low temperature coefficient modes with other modes that are sensitive to physical/environmental signals. It has been proved that the shear mode has a lower TCF than the other mode. Therefore, a low TCF resonant sensor can be developed by coupling shear mode with other modes. In 2013, Roozbeh Tabrizian et al. [110] developed a resonator, of which the extensional and shear mode are acoustically coupled through evanescent wave in the intermediate region. The central part $\left(W_{1}\right)$ of the sensor works in WE1 mode while the edge part $\left(W_{2}\right)$ resonates at the WS2 mode with the same frequency of central part. Results showed that compared with SiBAR, the WCRB sensor has achieved considerable $Q$ enhancement and TCF reduction.

Besides the low TCF characteristics, high sensitivity is another merit of WCRB sensor, which can be obtained by precisely deducing the beat frequency. After developing the shear-extensional mode coupled resonator, Roozbeh Tabrizian et al. developed a WCRB sensor for pressure measurement with high sensitivity in 2014 [111], as shown in Figure 16. In this sensor, 2 silicon bulk acoustic resonators (SiBAR) are excited in their 3rd lengthextensional mode $\left(\mathrm{LE}_{3}\right)$ and are acoustically coupled through thin vertical membranes. When out-of-phase bulk acoustic wave propagates into vertical membranes, the vertical membranes is excited to work at extensional Lamb mode as shown in Figure 17. Moreover, when in-phase bulk acoustic wave propagates into the vertical membranes, the vertical membranes is excited to work at transverse flexural resonant mode. The transverse flexural mode is sensitive to their surrounding air molecule while the extensional mode is not. Hence, a pressure sensor with an amplified sensitivity can be developed by coupling the two resonant modes. In such WCRB sensor, the beat frequency $f_{\mathrm{b}}$ is used to describe the pressure changes and can be expressed with:

$$
f_{b} \approx \frac{1}{2 \pi}\left|\sqrt{\frac{K_{\mathrm{LE}_{3}}+\alpha^{2} K_{f k}}{M_{\mathrm{LE}_{3}}+\alpha^{2}\left(M_{f l x}+\Delta M(P)\right)}}-\sqrt{\frac{K_{\mathrm{LE}_{3}}}{M_{\mathrm{LE}_{3}}}}\right|
$$

where $\Delta M(P)$ is the Pressure sensitive mass, $\alpha$ is the acoustic coupling efficiency, $\mathrm{M}_{\mathrm{i}}$ and $\mathrm{K}_{\mathrm{i}}\left(\mathrm{i} \in\left\{\mathrm{LE}_{3}, f l x\right\}\right)$ are the equivalent mass, spring of $\mathrm{LE}_{3}$ mode in SiBARs, and extensional and flexural modes in vertical membranes, respectively. In fact, the length of thin vertical membranes ( $2 \mathrm{~L}$ ) is 2 times than the length of SiBARs, which makes the $\mathrm{LE}_{3}$ mode of SiBARs and extensional Lamb mode of vertical membranes to resonate at the same frequency but a different wavelength. Results showed that a high sensitivity of $346 \mathrm{ppm} / \mathrm{kPa}$ has been achieved in the pressure range of $0-100 \mathrm{kPa}$, which is more than 500 times higher than that of pressure sensor in [6], whose sensitivity is $0.69 \mathrm{ppm} / \mathrm{kPa}$.

Comparisons of WCRB sensors are listed in Table 7. It can be observed that high sensitivity and low TCF can be obtained through proper acoustic coupling. Although WCRB sensors are currently only proof-of-concept, they have been proven to be promising for sensing applications and can be further enhanced by optimizing measurement and design methods. 


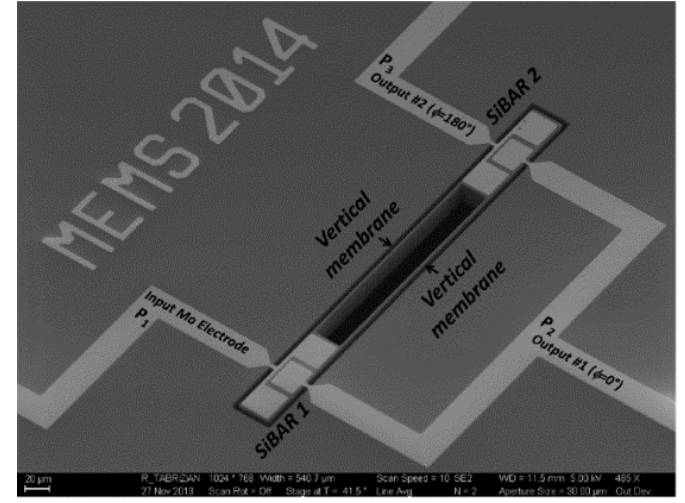

(a)

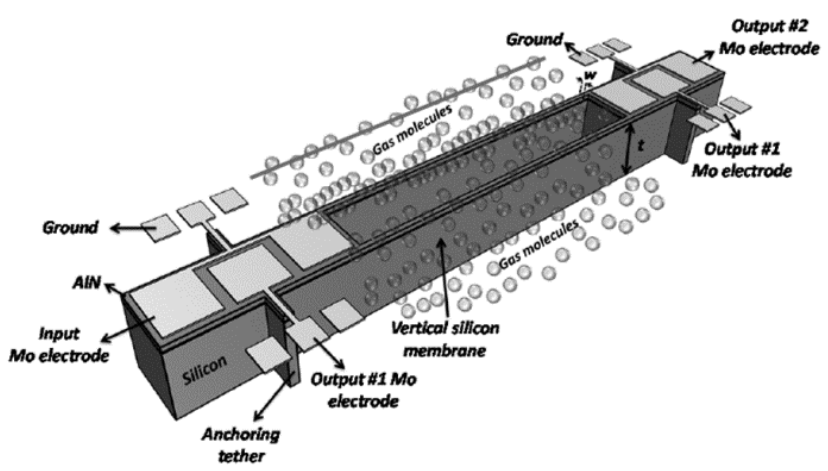

(b)

Figure 16. (a) SEM photo of device; (b) Schematic view of WCRB sensor for pressure measurement. Reproduced with permission from [111]. Copyright @ 2014, IEEE.

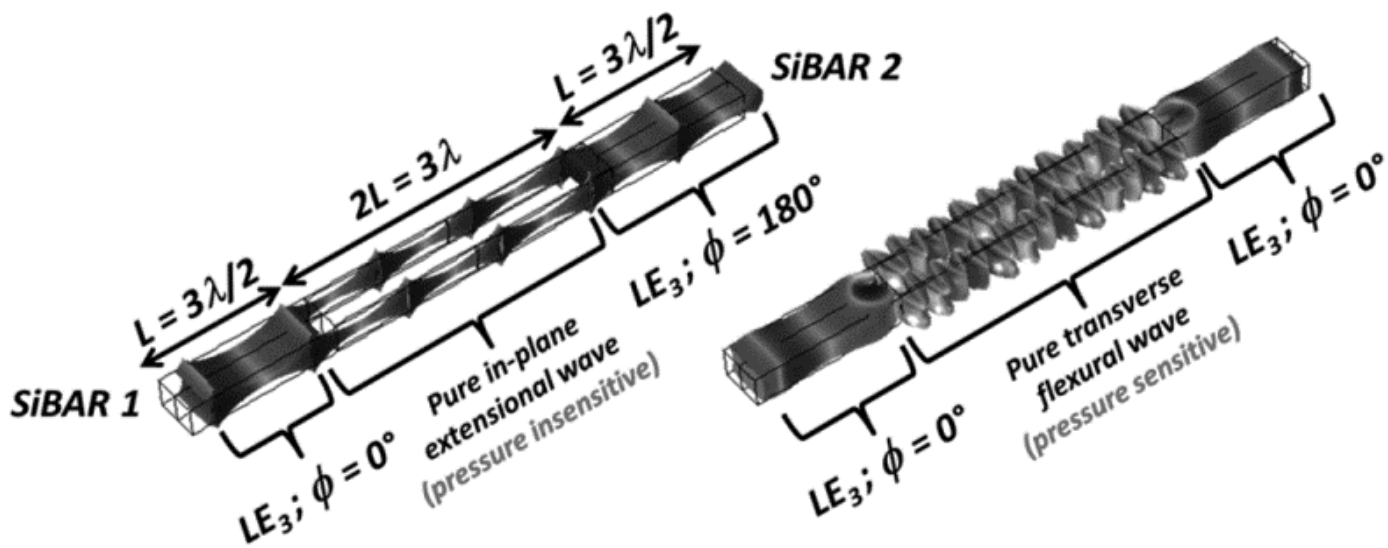

(a)

(b)

Figure 17. Mode shapes of (a) pressure insensitive extensional mode; (b) pressure sensitive flexural mode. Reproduced with permission from [111]. Copyright @ 2014, IEEE.

Table 7. Comparisons of properties of WCRB sensors.

\begin{tabular}{|c|c|c|c|}
\hline Reference & Coupling Way & Sensitivity & Merits \\
\hline [109] & $\begin{array}{l}\text { 3rd width-extensional mode (WE3) and cross-sectional } \\
\text { distortional mode }\end{array}$ & $-8300 \mathrm{ppm} /{ }^{\circ} \mathrm{C}$ & High temperature sensitivity \\
\hline [110] & $\begin{array}{l}\text { 1st width-extensional mode (WE1) and 2nd Width-shear } \\
\text { mode (WS2) }\end{array}$ & - & Q enhancement and TCF reduction \\
\hline [111] & $\begin{array}{c}\text { 3rd length-extensional mode (LE3) and transverse } \\
\text { flexural mode }\end{array}$ & $346 \mathrm{ppm} / \mathrm{kPa}$ & High pressure sensitivity \\
\hline
\end{tabular}

\subsection{UCRB Sensors}

UCRB sensors are the last type of DRB and MRB MEMS sensors. In fact, some UCRB sensors have two or more uncoupled resonators, while others have only one resonator. Those UCRB sensors can obtain the ability of high sensitivity and low TCF by properly controlling the resonators. On the basis of the spatial relationship of resonators, UCRB sensors can be mainly classified into three types: direct connection (DC-UCRB) sensors, quasi dual resonator (Q-UCRB) sensors and physically separated (PS-UCRB) sensors. 


\subsubsection{DC-UCRB Sensors}

The first type of UCRB sensors is the so-called DC-UCRB sensors. Although connected by physical structures, those resonators are not coupled to each other. One of the resonators is used as a sensor, while the other resonator is used as a reference. Study [112] has shown that based on the beat frequency of the two resonators, the DC-UCRB sensor can achieve excellent temperature sensing performance. Besides high sensitivity, the dualmode oscillator can realize self-temperature compensation and avoid the temperature hysteresis effect caused by the external temperature compensation sensor, which permits the realization of high-accuracy Microcomputer-Compensated Crystal Oscillators (MCXO).

Usually, a beat frequency $f_{\mathrm{B}}$ is used as the output and can be defined as:

$$
f_{\mathrm{B}}=m \times f_{1}-f_{2}
$$

where $m$ is the frequency multiplication factor, $f_{1}$ and $f_{2}$ are the first and the second resonant frequency, respectively. Normally, $f_{1}$ should be less than $f_{2}$. It has been proved that high sensitivity performance can be realized by designing the beat frequency to be as small as possible compared with the natural frequency of the resonator. Thus, it is best to make the value of $m$ as close as possible to the frequency ratio $r=f_{2} / f_{1}$.

In 2017, H. Campanella et al. [113] developed a DC-UCRB sensor for temperature sensing as shown in Figure 18b. It consists of two resonators, of which the frequencies are widely separated. The frequency of the outer resonator is $180 \mathrm{MHz}$ while that of the inner resonator is $500 \mathrm{MHz}$. Each resonator is excited in fundamental symmetric Lamb-wave modes (S0). Results showed that when the beat frequency is designed as $11.5 \mathrm{MHz}$, the $\mathrm{TCF}$ of the beat frequency $\left(-334 \mathrm{ppm} /{ }^{\circ} \mathrm{C}\right)$ is much larger than the separately extracted first-order TCFs of the outer $\left(-30 \mathrm{ppm} /{ }^{\circ} \mathrm{C}\right)$ and inner resonator $\left(-23 \mathrm{ppm} /{ }^{\circ} \mathrm{C}\right)$, which proved the UCRC sensor's suitability a for highly sensitive temperature sensor.

Generally, DC-UCRB sensors are promising in designing high-sensitivity sensors. However, due to the transverse wave propagation between two resonators, it has not become a hotspot in the research field.

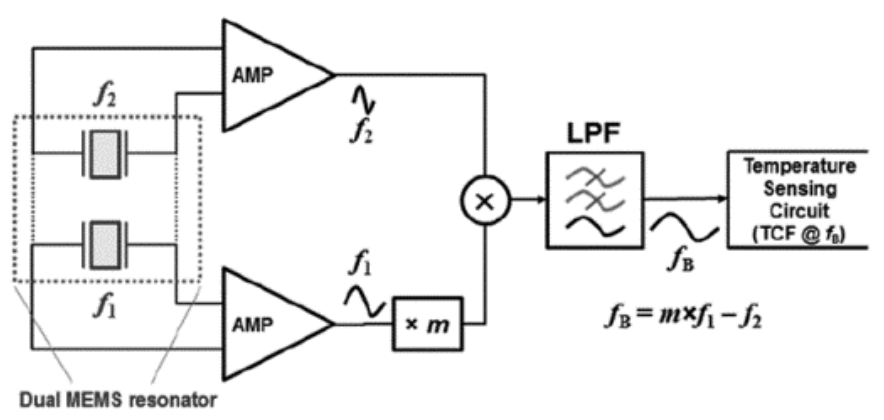

(a)

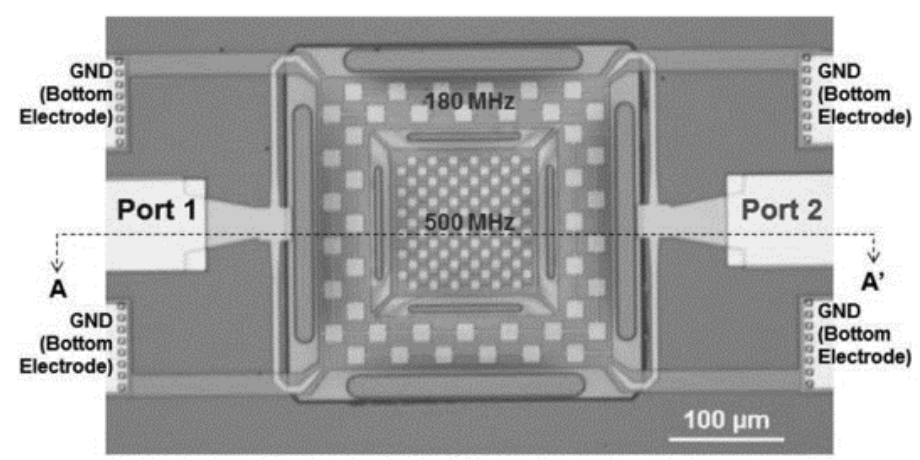

(b)

Figure 18. (a) schematic of beat frequency-based sensor system; (b) Optical picture of the concentric dual AIN MEMS Resonators. Reproduced with permission from [113]. Copyright (C) 2017, IEEE. 


\subsubsection{Q-UCRB Sensors}

The Q-UCRB sensors are the second kind of UCRB sensors. In fact, Q-UCRB sensors are essentially SRB sensors, with only one resonator working in dual or multiple modes [114-117] by properly controlling the excitation circuit. Although there is only one resonator, the single resonator working in dual modes can play the same role as dual resonators. Hence, the merits of dual resonators sensors can be inherited in Q-UCRB sensors. To ensure that the Q-UCRB sensor can work at dual modes, we should carefully design the excitation circuit on the basis of Barkhausen's criteria [119]. In addition, to achieve high sensitivity performance, it is necessary to carefully design the beat frequency calculation algorithm.

In 2011, the group of Farrokh Ayazi fabricated a Q-UCRB sensor for high-performance temperature sensing, of which the resonator vibrates at its fundamental $\left(f_{1}\right)$ and third-order $\left(f_{3}\right)$ length extensional modes as shown in Figure 19a [114]. The frequencies of $f_{1}$ and $f_{3}$ are $30 \mathrm{MHz}$ and $87 \mathrm{MHz}$, as shown in the top of Figure 20, respectively. When it is simultaneously driven by two loops (bottom left of Figure 20), the resonator will produce an output, which is a superposition of two signals. Results showed that a beat frequency $\left(f_{\mathrm{b}}\right)$ with a TCF of $162 \mathrm{ppm} /{ }^{\circ} \mathrm{C}$ was obtained. Three years later, they [115] further enhanced the performance of Q-UCRB sensor by simultaneously exciting the in-plane width-shear (WS) and width-extensional (WE) modes of the silicon resonator. Results showed that when the beat frequency is equal to $17 \mathrm{kHz}$, the highest TCF of the beat frequency is $1480 \mathrm{ppm} /{ }^{\circ} \mathrm{C}$, which is almost 10 times that of the Q-UCRB sensor developed in 2011.

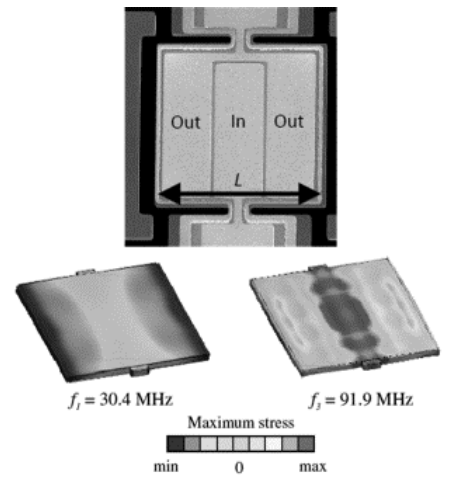

(a)

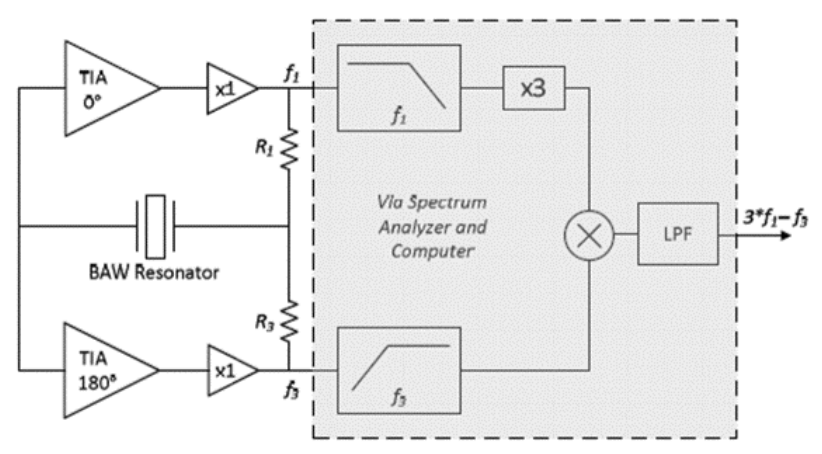

(b)

Figure 19. (a) SEM photo of device and the vibrating mode; (b) block diagram of Q-UCRB sensor. Reproduced with permission from [114]. Copyright @ 2011 , IEEE.
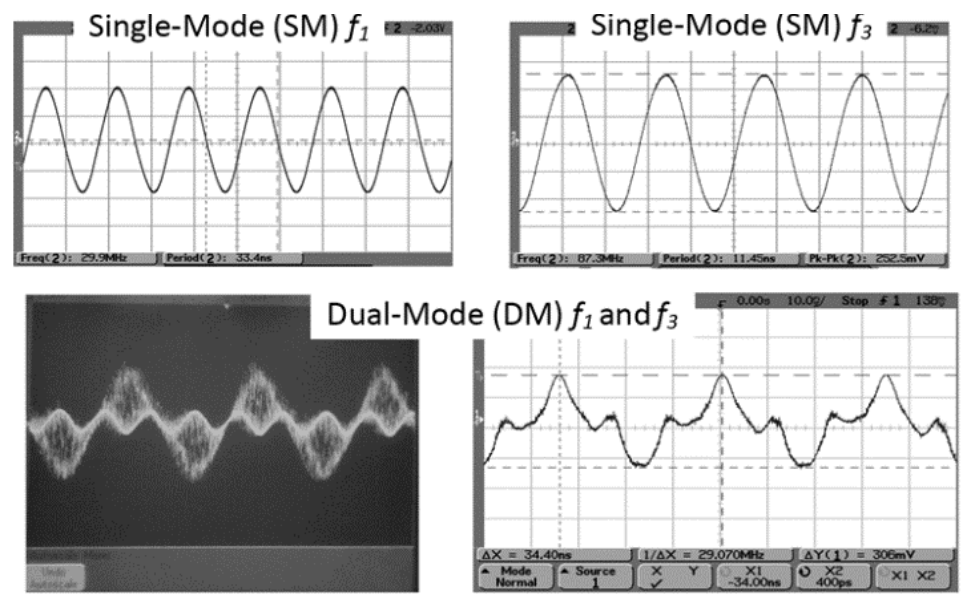

Figure 20. (top) Oscillator response for $f_{1}$ and $f_{3}$ single-mode excitation; (bottom left) dual-mode excitation at $f_{1}$ and $f_{3} ;$ (bottom right) dual-mode output signal after averaging. Reproduced with permission from [114]. Copyright $@$ 2011, IEEE. 
Besides the abovementioned works, they [109] developed another Q-UCRB sensor for temperature sensing in 2013. As shown in Figure 21, the central part of the resonator is designed to operate in the in-plane lamb wave mode. On the basis of the dispersion characteristic, the out-of-plane mode can also be excited in the central part by adjusting the width of the interdigital electrodes. Thus, the Q-UCRB sensor can run at multiple resonant modes simultaneously and independently. Particularly, the integer frequencies ratio of in-plane mode and out-of-plane mode can generate a small beat frequency and thus an amplified temperature sensitivity. Results showed that the TCF of beat frequency is $8292 \mathrm{ppm} /{ }^{\circ} \mathrm{C}$, which is a big advancement in comparison to the abovementioned work of this group.

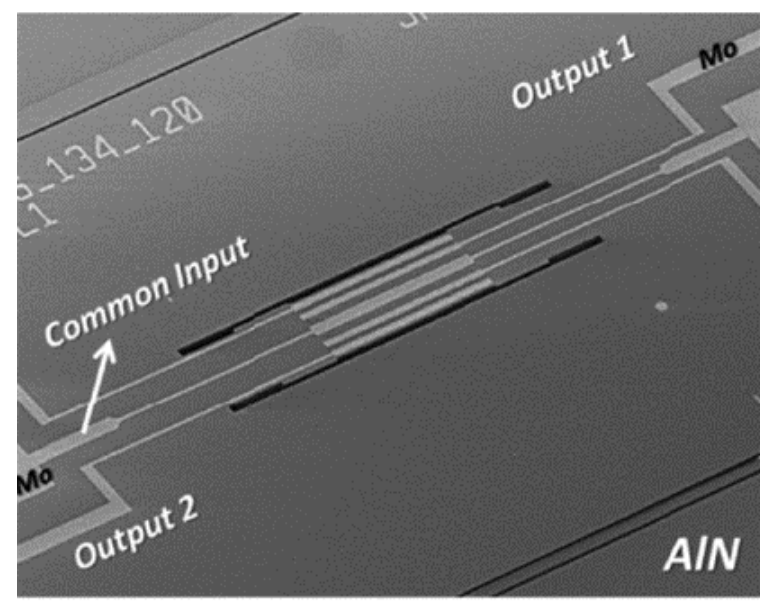

(a)

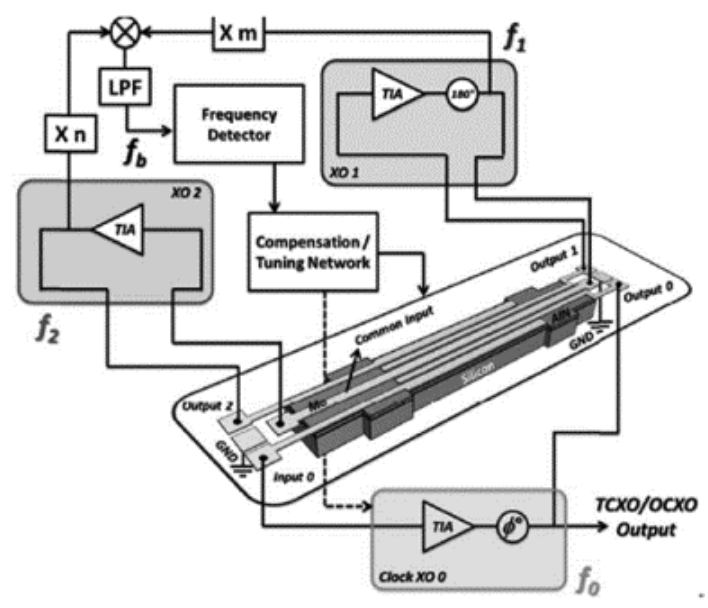

(b)

Figure 21. (a) SEM image; and (b) Schematic block diagram of a reference oscillator for Q-UCRB sensor for temperature sensing. Reproduced with permission from [109]. Copyright (C) 2013, IEEE.

Other groups have also carried out research on the Q-UCRB sensor. In 2012, L. Garcia-Gancedo et al. [116] developed a dual-mode thin film bulk acoustic wave resonator (FBAR) which can precisely measure the mass loading and the temperature in parallel, without the need for additional reference devices or complicated electronics for real-time temperature compensation. In 2018, Congcong Gu et al. [117] proposed a dual-mode film bulk acoustic resonator (FBAR) based pressure sensor. The feasibility of on-chip temperature compensation of such sensor has been proved by the experimental results. However, it still cannot be used in practical applications because it is difficult to design a circuit to enable the sensor to simultaneously work in two resonant modes.

The comparisons of different Q-UCRB sensors are listed in Table 8. We can draw the conclusion that Q-UCRB sensors usually have high sensitivity and can realize temperature compensation without external reference. More importantly, they can detect multiple parameters simultaneously.

Table 8. Comparisons of Q-UCRB sensors.

\begin{tabular}{ccc}
\hline Sensor & Mode & Output \\
\hline Temperature sensor [114] & $\begin{array}{c}\text { Fundamental }\left(f_{1}\right) \text { and third-order }\left(f_{3}\right) \text { length } \\
\text { extensional mode }\end{array}$ & TCF 162 ppm $/{ }^{\circ} \mathrm{C}$ \\
\hline Temperature sensor [115] & $\begin{array}{c}\text { In-plane width-shear }(\mathrm{WS}) \text { and } \\
\text { width-extensional }(\mathrm{WE}) \text { modes }\end{array}$ & TCF 1480 ppm $/{ }^{\circ} \mathrm{C}$ \\
\hline Temperature sensor [109] & in-plane and out-of-plane lamb wave mode & TCF 8292 $\mathrm{ppm} /{ }^{\circ} \mathrm{C}$ \\
\hline Temperature and mass-loading sensor [116] & FBAR & Mass loading and the temperature \\
\hline Pressure sensor [117] & FBAR & $\begin{array}{c}\text { Mode } 1: 1.642 \mathrm{ppm}(\mathrm{kPa})^{-1} \\
\text { Mode 2: } 0.1764 \mathrm{ppm}(\mathrm{kPa})\end{array}$ \\
\hline
\end{tabular}




\subsubsection{PS-UCRB Sensors}

The last type of UCRB sensors is PS-UCRB sensors [118-120]. Resonators of PS-UCRB sensors are separately located in the same environment and will drift in the same or inverse directions in response to changes through appropriate design. The temperature-induced resonant frequency shift needs to be suppressed by feasible algorithm or hardware.

In 2015, the group of Junbo Wang [118] developed a PS-UCRB sensor for pressure measurement with the ability of self-temperature compensation. As shown in Figure 22, there are two " $\mathrm{H}$ "-shaped double-clamped beams, which are physically separated by gaps, named as central beam and side beam. With almost identical dimensions and comparable resonant frequencies, the resonant frequencies of those two beams drifted in the same direction in response to temperature changes. Experimental results showed that the pressure sensor has good properties with error less than $\pm 0.01 \%$ of the full pressure scale $(50 \mathrm{kPa} \sim 110 \mathrm{kPa})$ in the entire temperature range $\left(-40^{\circ} \mathrm{C} \sim 70^{\circ} \mathrm{C}\right)$.

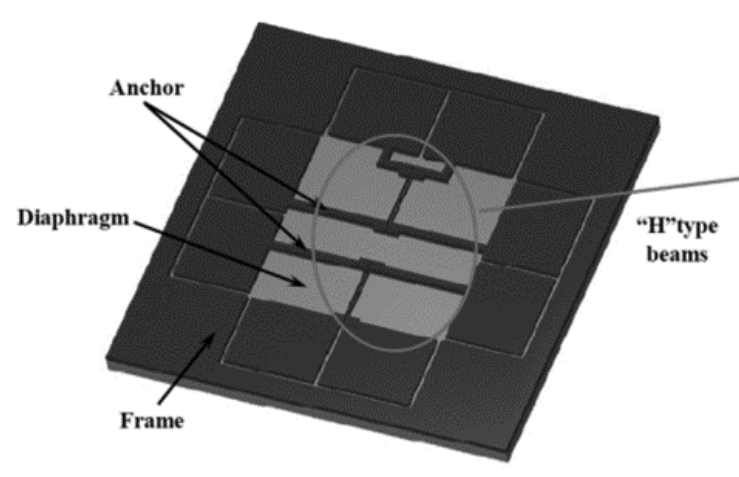

(a)

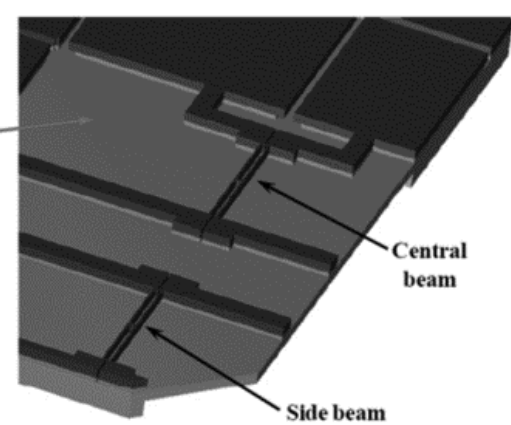

(b)

Figure 22. (a) Overall and (b) Partial enlarged SEM schematic of pressure sensors. Reproduced with permission from [118].

In 2017, Qingyun Xie et al. [119] developed a PS-UCRB sensor for high pressure measurement in high temperature environment as shown in Figure 23. Two methods are adopted to reduce the temperature influence: the first is to make an oxide trench array (OTA) in the poly-Si layer, and the second is to design algorithm based on dual-frequency resonating device. It was reported that a high-pressure sensor with a non-linearity of $2.28 \%$ F.S. has been obtained over a wide temperature range of $360^{\circ} \mathrm{C}$.
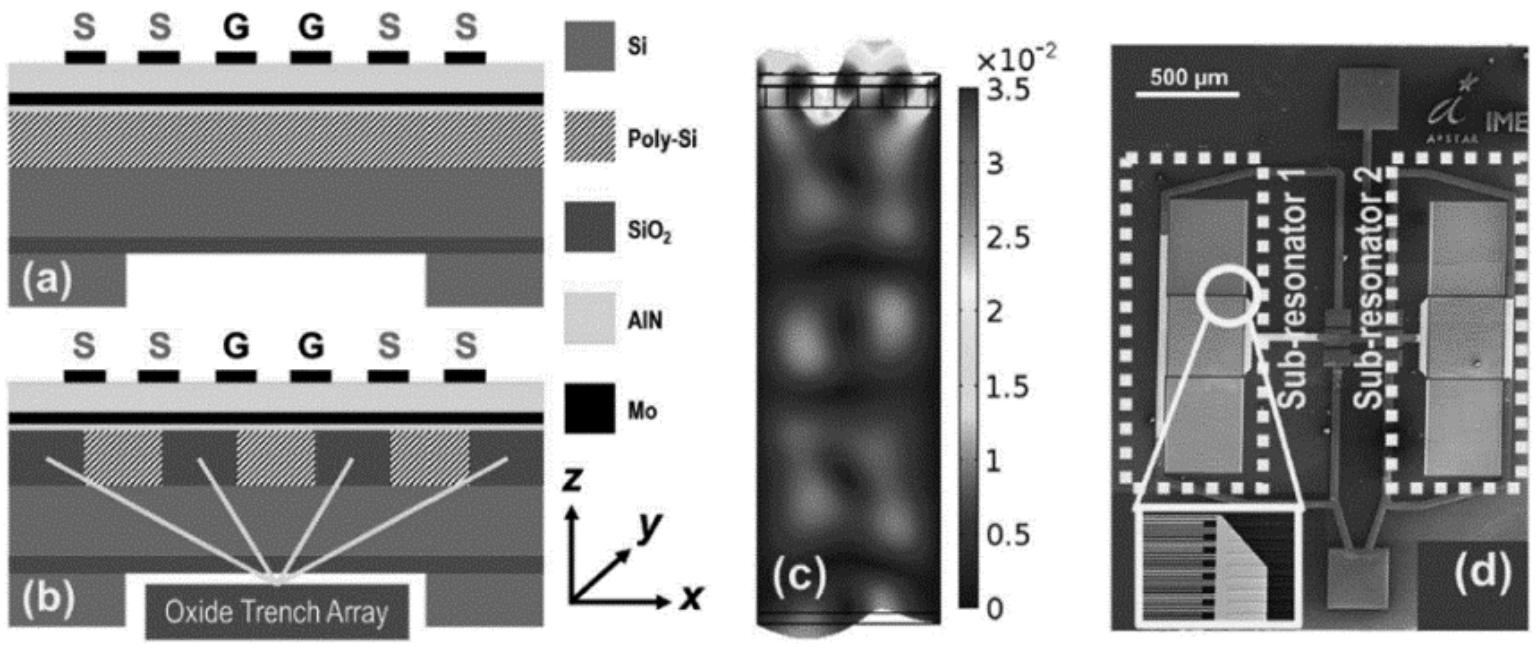

Figure 23. Dual-frequency resonating device (a) resonator without OTA; (b) resonator with OTA; (c) Displacement of the quasi-SAW mode shape of resonator; (d) SEM image of device. Reproduced with permission from [119]. Copyright (C) 2017, IEEE. 
In the abovementioned PS-UCRB sensor, the temperature-induced resonant frequency shift is suppressed by algorithm. In addition to temperature compensation algorithms, temperature compensation can also be implemented through hardware. SiTime Company [120] developed a high-resolution PS-UCRB sensor for temperature measurement (Figure 24a), of which the temperature information is deduced by hardware. It is shown that two pairs of four-ring-resonator structure are designed with inverse response and different sensitivity of temperature. A hardware-based approach of measuring the frequency ratio of two on-chip four-ring-resonator structure (Figure 24b) is designed [121,122] to generate high temperature resolution. The output frequency can be expressed by:

$$
f_{\text {out }}=[(1+T D C) \times P F M] \times f_{\text {ref }} / N_{\text {div }}
$$

where $f_{\text {out }}$ and $f_{\text {ref }}$ are the frequencies of output and PLL reference clock frequency, respectively. TDC is the frequency ratio of two on-chip resonators, PFM is the programmable frequency multiplier, and $N_{\text {div }}$ is the value of divider.

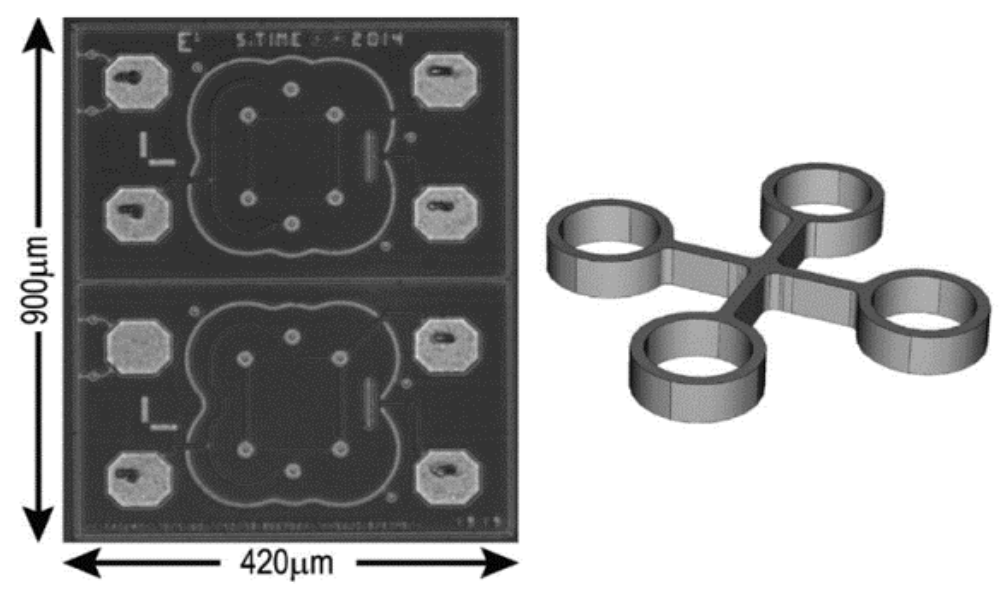

(a)

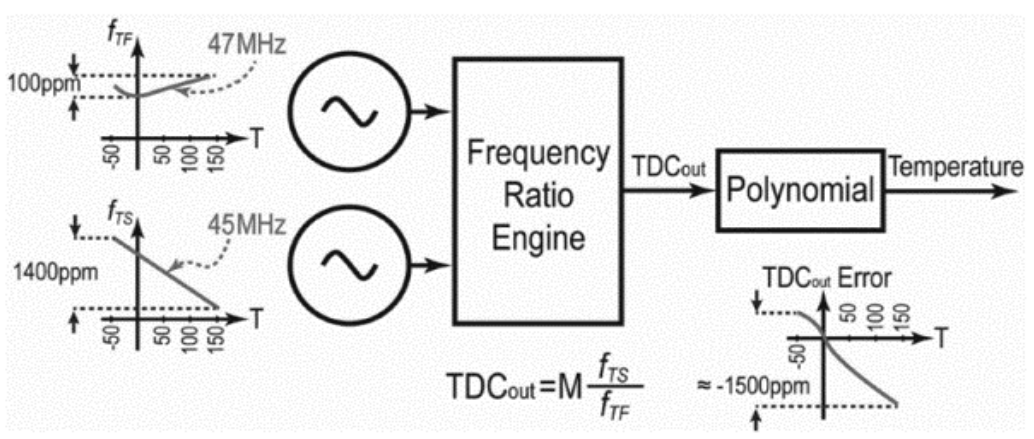

(b)

Figure 24. (a) 3-D view of MEMS resonators and the MEMS dies photo; (b) Architecture of a temperature sensor operating based on measuring the ratio of the frequencies. Reproduced with permission from [120]. Copyright (C) 2017, IEEE.

The block diagram of temperature measurement with the PS-UCRB sensor is shown in Figure 25. The first resonator operates at $47 \mathrm{MHz}$ which exhibits a less than $\pm 50 \mathrm{ppm}$ frequency stability in the temperature range of $-45^{\circ} \mathrm{C}$ to $105^{\circ} \mathrm{C}$. The second resonator oscillates at $45 \mathrm{MHz}$ with a temperature coefficient of $-7 \mathrm{ppm} / \mathrm{K}$. Results showed that the PS-UCRB sensor have a very high resolution of $20 \mu \mathrm{K}$ over a bandwidth of $100 \mathrm{~Hz}$. Now, this work has been successfully applied to the suppression of temperature-caused resonant frequency shift in commercialized timer chips. 


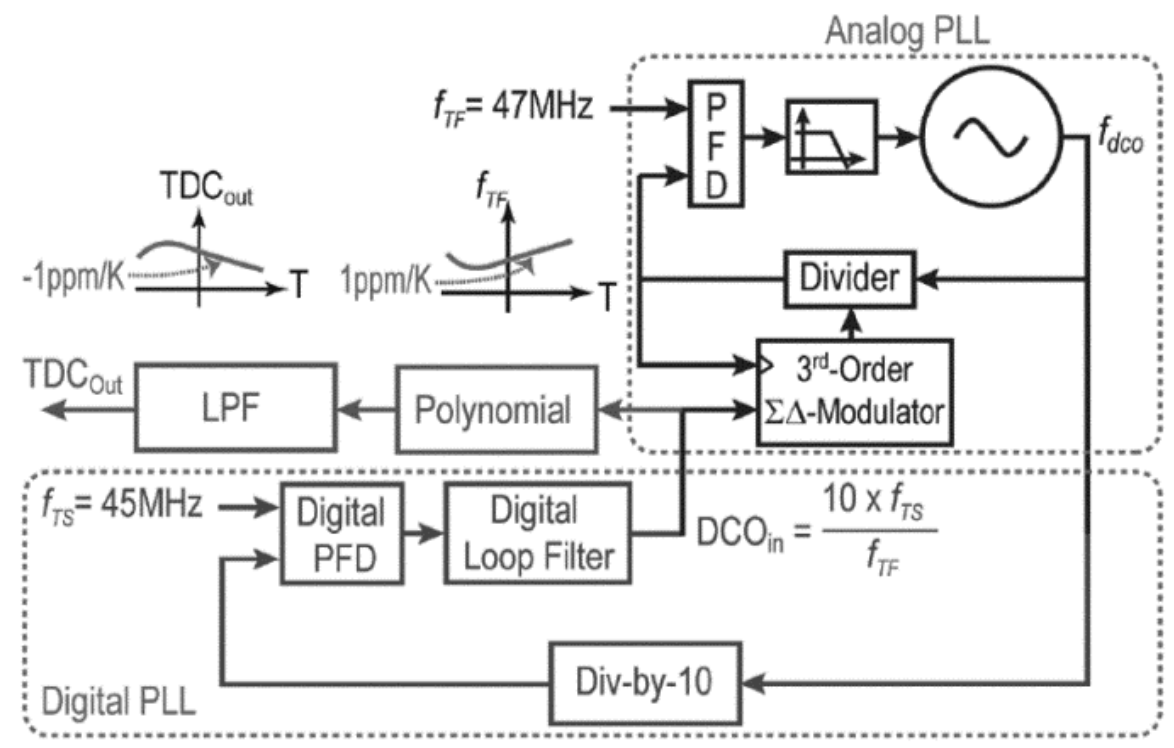

Figure 25. Block diagram of temperature measurement with the PS-UCRB sensor. Reproduced with permission from [120]. Copyright (C) 2017, IEEE.

The comparisons of different PS-UCRB sensors are listed in Table 9. We can see that high resolution and low TCF can be obtained. The virtues of PS-UCRB sensors are as follows: (1) using two separated resonators for good temperature compensation and (2) high resolution of temperature sensing.

Table 9. Comparisons of PS-UCRB sensor.

\begin{tabular}{ccc}
\hline Sensor & $\begin{array}{c}\text { Temperature Compensation } \\
\text { Method }\end{array}$ & Output \\
\hline Pressure sensor [118] & Algorithm compensation & Low error less than $\pm 0.01 \%$ \\
\hline High pressure sensor [119] & $\begin{array}{c}\text { Algorithm compensationand } \\
\text { material matching }\end{array}$ & Non-linearity of $2.28 \%$ F.S \\
\hline Temperature sensor [120] & Hardware deducing & High resolution of $20 \mu \mathrm{K}$ \\
\hline
\end{tabular}

\subsubsection{Summary}

In this section, three different types UCRB sensors, named as the DC-UCRB sensor, the Q-UCRB sensor and the PS-UCRB sensor, were introduced in detail, respectively. In general, these UCRB sensors have the merits of high sensitivity, parallelize measurement, and common change rejection. Yet, there are still shortcomings that need to be resolved, such as how to simultaneously excite multiple oscillator circuits and how to optimize the signal-to-noise ratio and the linearity of the beat frequency.

\section{Conclusions}

Dual-Resonator-Based (DRB) and Multiple-Resonator-Based MEMS Sensors are comprehensively and critically reviewed in this paper. The review started with understanding the principle of single resonator and followed by the analysis of coupled resonators based on the principle of mode-localization. Merits of sensitivity enhancement of coupled resonator using vibration amplitude ration (AR) as output compared with frequency shift output are highlighted. Following the analysis of coupled resonators, three different classes DRB and MRB MEMS sensors, named strength-coupled-resonator-based (SCRB) sensors, wave-coupled-resonator-based (WCRB) sensors, and uncoupled-resonator-based (URB) sensors are introduced and analyzed in detail, respectively. Several sensing applications, such as mass sensor, displacement sensor, electrometer, accelerometer, stiffness sensor, and force sensor are given. A comparative performance between DRB and MRB sensors and 
single-resonator-based sensors are also provided in tables to demonstrate their advantages of sensitivity enhancement. Studies revealed that weaker effective coupling (lower $k$ ) between the resonators can help further enhance the sensitivity of such sensors. Besides high sensitivity, the ability of common mode rejection is another advantage of DRB and MRB MEMS sensors, which are also analyzed throughout the study. Results showed that AR has an excellent disturbance suppression capability compared to the frequency shift output. Following the analysis of common mode rejection, another sensor's performance characterization, resolution, are discussed and analyzed. Regarding whether the resolution of the mode local sensor is related to the coupling coefficient $k$, there are mainly two different views. Anyhow, the resolution of mode-localized sensors is related to multiple factors such as sensitivity, noise, and quality factors, we can improve the resolution by further increasing the sensitivity and reducing the amplitude noises both of extrinsic noise arising from the external electronic interfacial readout circuitry, and intrinsic noises that are inherent to the micro- or nanomechanical resonator arrays.

Overall, DRB and MRB MEMS sensors have many advantages over SRB sensors, such as high sensitivity and common mode rejection. These advantages make them attractive for many applications in high-end markets, such as the measurement of ultra-small mass sensors and ultra-low accelerometers. This review assessment is proposed to serve as a one-stop reference for other researchers in the field to access a comprehensive outlook on the promising DRB and MRB MEMS sensors.

\section{Future Perspectives of DRB and MRB Sensors}

Owing to their inherent merits of high sensitivity and common mode rejection, DRB and MRB MEMS sensors are promising in future high-end markets. However, a lot of work still needs to be conducted to bridge the gap between sensor research and market applications, especially in the following two aspects.

Firstly, fabrication processes are the key factor in the realization of DRB and MRB MEMS sensors, which need to be further optimized. For SCRB sensor, the realization of high stability and high $\mathrm{Q}$ factor resonators depends on the degree of sealing of the device in a vacuum environment. The better the vacuum environment, the higher of the quality factor. Obviously, based on the Leeson model [135], a high $Q$ is very important to reduce the phase noise level of the resonator. For the WCRB sensor, precise fabrication of the structure is the key factor to complete the coupling of different dimension-related resonant mode. Moreover, it can also further reduce the leakage of acoustic wave from the edge of the resonant structure to greatly improve the precision of resonant frequency, and ultimately enhance the stability of frequency. For the DC-UCRB and the Q-UCRB sensor, high fabrication precision determines the sensitivity and the linearity of beat frequency due to the piezoelectric transduction principle. For the PS-UCRB sensor, the fabrication of same resonant structure is the most important factor, which is key in obtaining high sensitivity. Therefore, the manufacturing process needs to be further improved in the future.

Secondly, the control circuit needs to be further studied. The groups of Honglong Chang [73] and A. A. Seshia [136] have contributed a lot for the self-oscillating circuit loop of SCRB sensor. On the basis of these developed self-oscillating closed loops, the SCRB sensors have achieved the abilities of high sensitivity, high resolution, and common mode rejection. Especially, the high signal-to-ratio noise (124.2 dB) of self-oscillating closed loop has been obtained by group of Honglong Chang. Such work has greatly improved the development of DRB and MRB MEMS sensors. Besides, the frequency stability needs to be further enhanced. So far, the best frequency stability achieved by A. A. Seshia group is $7.5 \mathrm{ppb}$. There are some effective ways to improve frequency stability [136], such as the optimal operating point of the oscillator, low power consumption and integrated implementation of the oscillator, as well as integration with a temperature compensation scheme. For WCRB and UCRB sensors, besides the PS-UCRB sensor, the optimization of signal-to-ratio noise and the linearity of beat frequency is the imperative work. For 
the PS-UCRB, the optimization of circuit should be conducted by combining the design of algorithm.

All in all, dual-resonator-based (DRB) and multiple-resonator-based (MRB) sensors are indeed promising in future high-end markets, but there is still a long way to go before realizing their practical applications in the field of sensing.

Funding: This research was funded by National Natural Science Foundation of China, grant number 61871363.

Conflicts of Interest: The authors declare no conflict of interest.

\section{References}

1. Stemme, G. Resonant silicon sensors. J. Micromech. Microeng. 1991, 1, 113. [CrossRef]

2. Shi, X.; Lu, Y.; Xie, B.; Xiang, C.; Wang, J.; Chen, D. A Double-Ended Tuning Fork Based Resonant Pressure Micro-Sensor Relying on Electrostatic Excitation and Piezoresistive Detection. Proceedings 2018, 2, 875. [CrossRef]

3. Shi, X.; Lu, Y.; Xie, B.; Xiang, C.; Wang, J.; Chen, D. A Resonant Pressure Microsensor Based on Double-Ended Tuning Fork and Electrostatic Excitation/Piezoresistive Detection. Sensors 2018, 18, 2494. [CrossRef] [PubMed]

4. Xie, B.; Xing, Y.; Wang, Y.; Chen, J.; Chen, D.; Wang, J. A Lateral Differential Resonant Pressure Micro Sensor Based on SOI-Glass Wafer-Level Vacuum Packaging. Sensors 2015, 15, 24257-24268. [CrossRef] [PubMed]

5. Xie, B.; Xing, Y.; Wang, Y.; Wang, J.; Chen, D. Vacuum-packaged Resonant Pressure Sensor with Dual Resonators for High Sensitivity and Linearity. Procedia Eng. 2015, 120, 194-199. [CrossRef]

6. Zhang, M.; Zhao, Z.; Du, L.; Fang, Z. A film bulk acoustic resonator-based high-performance pressure sensor integrated with temperature control system. J. Micromech. Microeng. 2017, 27, 045004. [CrossRef]

7. Yantchev, V.; Katardjiev, I. Thin film Lamb wave resonators in frequency control and sensing applications: A review. J. Micromech. Microeng 2013, 23, 043001. [CrossRef]

8. Anderas, E.; Katardjiev, I.; Yantchev, V. Lamb wave resonant pressure micro-sensor utilizing a thin-film aluminum nitride membrane. J. Micromech. Microeng. 2011, 21, 085010. [CrossRef]

9. Bernstein, J.; Cho, S.; King, A.T.; Kourepenis, A.; Maciel, P.; Weinberg, M. A micromachined comb-drive tuning fork rate gyroscope. In Proceedings of the International Conference on Micro Electro Mechanical Systems, Fort Lauderdale, FL, USA, 10 February 1993; pp. 143-148. [CrossRef]

10. Liang, W.; Ilchenko, V.S.; Savchenkov, A.; Dale, E.; Eliyahu, D.; Matsko, A.B.; Maleki, L. Resonant microphotonic gyroscope. Optica 2017, 4, 114-117. [CrossRef]

11. Norouzpour-Shirazi, A.; Zaman, M.F.; Ayazi, F. A Digital Phase Demodulation Technique for Resonant MEMS Gyroscopes. IEEE Sens. J. 2014, 14, 3260-3266. [CrossRef]

12. Johari, H.; Ayazi, F. Capacitive bulk acoustic wave silicon disk gyroscopes. In Proceedings of the International Electron Devices Meeting, San Francisco, CA, USA, 11-13 December 2006; pp. 1-4. [CrossRef]

13. Guo, Z.; Cheng, F.; Li, B.; Cao, L.; Lu, C.; Song, K. Research development of silicon MEMS gyroscopes: A review. Microsyst. Technol.-Micro-Nano-Syst.-Inf. Storage Process. Syst. 2015, 21, 2053-2066. [CrossRef]

14. Tabrizian, R.; Hodjat-Shamami, M.; Ayazi, F. High-Frequency AlN-on-Silicon Resonant Square Gyroscopes. J. Microelectromech. Syst. 2013, 22, 1007-1009. [CrossRef]

15. Wang, S.; Wei, X.; Zhao, Y.; Jiang, Z.; Shen, Y. A MEMS resonant accelerometer for low-frequency vibration detection. Sens. Actuators A Phys. 2018, 283, 151-158. [CrossRef]

16. Zhang, J.; Su, Y.; Shi, Q.; Qiu, A. Microelectromechanical Resonant Accelerometer Designed with a High Sensitivity. Sensors 2015, 15, 30293-30310. [CrossRef]

17. Christensen, D.L.; Ahn, C.H.; Hong, V.; Ng, E.J.; Yang, Y.; Lee, B.; Kenny, T.W. Hermetically encapsulated differential resonant accelerometer. In Proceedings of the International Conference on Solid-State Sensors, Actuators and Microsystems 2013, Barcelona, Spain, 16-20 June 2013; pp. 606-609. [CrossRef]

18. Du, L.; Fang, Z.; Yan, J.; Zhao, Z. Enabling a wind energy harvester based on $\mathrm{ZnO}$ thin film as the building skin. Sens. Actuators A 2017, 260, 35-44. [CrossRef]

19. Fritsch, H.; Lucklum, R.; Iwert, T.; Hauptmann, P.; Scholz, D.; Peiner, E.A. Schlachetzki, A low-frequency micromechanical resonant vibration sensor for wear monitoring. Sens. Actuators A-Phys. 1997, 62, 616-620. [CrossRef]

20. Wang, P.; Tanaka, K.; Sugiyama, S.; Dai, X.; Zhao, X.; Liu, J. A micro electromagnetic low level vibration energy harvester based on MEMS technology. Microsyst. Technol.-Micro-Nanosyst.-Inf. Storage Process. Syst. 2009, 15, 941-951. [CrossRef]

21. Rugar, D.; Budakian, R.; Mamin, H.; Chui, B. Single spin detection by magnetic resonance force microscopy. Nature 2004, 430, 329-332. [CrossRef]

22. Mamin, H.; Rugar, D. Sub-attonewton force detection at millikelvin temperatures. Appl. Phys. Lett. 2001, 79, 3358-3360. [CrossRef]

23. Zhu, Y.; Lee, J.E.; Seshia, A. A resonant micromachined electrostatic charge sensor. IEEE Sens. J. 2008, 8, 1499-1505. [CrossRef]

24. Zhang, M.; Du, L.; Fang, Z.; Zhao, Z. A Sensitivity-Enhanced Film Bulk Acoustic Resonator Gas Sensor with an Oscillator Circuit and Its Detection Application. Micromachines 2017, 8, 25. [CrossRef] 
25. Johar, A.K.; Sharma, G.K.; Kumar, T.B.; Varma, T.; Periasamy, C.; Agarwal, A.; Boolchandani, D. Optimization of a flexible film bulk acoustic resonator-based toluene gas sensor. J. Electron. Mater. 2021, 50, 5387-5395. [CrossRef]

26. Liu, J.; Zhao, Z.; Fang, Z.; Liu, Z.; Zhu, Y.; Du, L. High-performance FBAR humidity sensor based on the PI film as the multifunctional layer. Sens. Actuators B Chem. 2020, 308, 127694. [CrossRef]

27. Zhou, L.; Manceau, J.; Bastien, F. Interaction between gas flow and a Lamb waves based microsensor. Sens. Actuators A 2012, 181, 1-5. [CrossRef]

28. Howe, R.T.; Muller, R.S. Resonant micro-bridge vapor sensor. IEEE Trans. Electron Devices 1986, 33, 499-506. [CrossRef]

29. Kong, H.; Lib, C.; Guo, Z.; Zhang, W.; Yao, J.; Zhu, H.; Yan, R.; Wang, L.; Li, J.; Wei, W.; et al. Sensitivity improved with Parylene-C passivized on Lamb wave sensor for aPTT measurement through monitoring whole blood reaction. Sens. Actuators B Chem. 2019, 285, 479-486. [CrossRef]

30. DeMiguel-Ramos, M.; Díaz-Durán, B.; Escolano, J.; Barba, M.; Mirea, T.; Olivares, J.; Clement, M.; Iborra, E. Gravimetric biosensor based on a 1.3 GHz AlN shear-mode solidly mounted resonator. Sens. Actuators B 2017, 239, 1282-1288. [CrossRef]

31. Liao, H.S.; Huang, K.Y.; Chang, C.S. Cantilever-based mass sensor using high order resonances for liquid environment. In Proceedings of the International Conference on Advanced Intelligent Mechatronics, Budapest, Hungary, 3-7 July 2011; pp. 652-655. [CrossRef]

32. Park, K.; Kim, N.; Morisette, D.T.; Aluru, N.; Bashir, R. Resonant MEMS mass sensors for measurement of micro-droplet evaporation. J. Microelectromech. Syst. 2012, 21, 702-711. [CrossRef]

33. Siwak, N.; Fan, X.; Goldsman, N.; Ghodssi, R. Indium phosphide resonant chemical sensor with a monolithically integrated optical readout scheme. In Proceedings of the IEEE SENSORS, Atlanta, GA, USA, 28-31 October 2007; pp. 1428-1431. [CrossRef]

34. Lee, H.J.; Park, K.K.; Oralkan, O.; Kupnik, M.; Khuri-Yakub, B.T. A multichannel oscillator for a resonant chemical sensor system. IEEE Trans. Ind. Electron. 2014, 61, 5632-5640. [CrossRef]

35. Jensen, K.; Kim, K.; Zettl, A. An atomic-resolution nano-mechanical mass sensor. Nat. Nanotechnol. 2008, 3, 533-537. [CrossRef]

36. Alam, S.; Mittal, U.; Islam, T. The oxide film coated surface acoustic wave resonators for the measurement of relative humidity. IEEE Trans. Instrum. Meas. 2021, 77, 1-9. [CrossRef]

37. Wang, J.; Park, M.; Mertin, S.; Pensala, T.; Ansari, A. A film bulk acoustic resonator based on ferroelectric aluminum scandium nitride films. J. Microelectromech. Syst. 2020, 29, 741-747. [CrossRef]

38. Gao, F.; Al-Qahtani, A.M.; Khelif, A.; Boussaid, F.; Bermak, A. Towards acoustic radiation free lamb wave resonators for high-resolution gravimetric biosensing. IEEE Sens. J. 2020, 21, 2725-2733. [CrossRef]

39. Jin, D.; Liu, J.; Li, X.; Zuo, G.; Wang, Y.; Yu, H.; Ge, X. Tens femtogram resoluble piezoresistive cantilever sensors with optimized high-mode resonance excitation. Proceedings of 1st IEEE International Conference on Nano Micro Engineered and Molecular Systems, 1st IEEE-NEMS 2006, Zhuhai, China, 18-21 January 2006; pp. 832-836. [CrossRef]

40. Zou, X.; Thiruvenkatanathan, P.; Seshia, A.A. A high resolution micro-electro-mechanical resonant tilt sensor. Sens. Actuators A Phys. 2014, 220, 168-177. [CrossRef]

41. Zou, X.; Thiruvenkatanathan, P.; Seshia, A.A. Micro-electromechanical resonant tilt sensor with 250 nano-radian resolution. In Proceedings of the 2013 Joint European Frequency and Time Forum and International Frequency Control Symposium, EFTF/IFC 2013, Prague, Czech Republic, 21-25 July 2013; pp. 54-57. [CrossRef]

42. Gupta, A.; Akin, D.; Bashir, R. Single virus particle mass detection using microresonators with nanoscale thickness. Appl. Phys. Lett. 2004, 84, 1976. [CrossRef]

43. Sone, H.; Okano, H.; Hosaka, S. Picogram mass sensor using piezoresistive cantilever for biosensor. Jpn. J. Appl. Phys. 2004, 43, 4663. [CrossRef]

44. Ilic, B.; Czaplewski, D.; Zalalutdinov, M.; Craighead, H.G. Single cell detection with micromechanical oscillators. J. Vac. Sci. Technol. B Microelectron. Nanom. Struct. 2001, 19, 2825-2828. [CrossRef]

45. Baek, I.; Byun, S.; Lee, B.; Ryu, J.; Kim, Y.; Yoon, Y.; Jang, W.; Lee, S.; Yu, H. Attogram mass sensing based on silicon micro-beam resonators. Sci. Rep. 2017, 7, 46660. [CrossRef]

46. Davis, Z.J.; Boisen, A. Aluminum nanocantilevers for high sensitivity mass sensors. Appl. Phys. Lett. 2005, 87, 013102. [CrossRef]

47. Ekinci, K.L.; Huang, X.M.H.; Roukes, M.L. Ultrasensitive nanoelectromechanical mass detection. Appl. Phys. Lett. 2004, 84, 4469-4471. [CrossRef]

48. Ono, T.; Esashi, M. Magnetic force and optical force sensing with ultrathin silicon resonator. Rev. Sci. Instrum. 2003, 74, 5141-5146. [CrossRef]

49. Abdolvand, R.; Bahreyni, B.; Lee, J.E.Y.; Nabki, F. Micromachined resonators: A review. Micromachines 2016, 7, 160. [CrossRef]

50. Wang, S.; Chen, W.; Bahr, B.; Fang, W.; Li, S.; Weinstein, D. Temperature coefficient of frequency modeling for CMOS-MEMS bulk mode composite resonators. IEEE Trans. Ultrason. Ferroelectr. Freq. Control 2015, 62, 1166-1178. [CrossRef]

51. Zhang, S.; Sahin, H.; Torun, E.; Peeters, F.; Martien, D.; DaPron, T.; Dilley, N.; Newman, N. Fundamental mechanisms responsible for the temperature coefficient of resonant frequency in microwave dielectric ceramics. J. Am. Ceram. Soc. 2017, 100, $1508-1516$. [CrossRef]

52. Boom, B.; Bertolini, A.; Hennes, E.; Brookhuis, R.; Wiegerink, R.; Brand, J.; Beker, M.; Oner, A.; Wees, D. Nano-G accelerometer using geometric anti-springs. In Proceedings of the International Conference on Micro Electro Mechanical Systems 2017, Las Vegas, VA, USA, 22-26 January 2017; pp. 33-36. [CrossRef] 
53. Gil-Santos, E.; Ramos, D.; Jana, A.; Calleja, M.; Raman, A.; Tamayo, J. Mass Sensing Based on Deterministic and Stochastic Responses of Elastically Coupled Nanocantilevers. Nano Lett. 2009, 9, 4122-4127. [CrossRef]

54. Saad, N.H.; Anthony, C.J.; Al-Dadah, R.; Ward, M.C. Exploitation of multiple sensor arrays in electronic nose. In Proceedings of the IEEE SENSORS, Christchurch, New Zealand, 25-28 October 2009; pp. 1575-1579. [CrossRef]

55. Hajhashemi, M. Characterization of Strongly Coupled Micro-Resonator Systems for Multi-Sensor Applications. Ph.D. Thesis, Simon Fraser University, Burnaby, BC, Canada, 2014. Available online: http://summit.sfu.ca/item/14701 (accessed on 13 November 2014).

56. Spletzer, M.; Raman, A.; Wu, A.Q.; Xu, X.; Reifenberger, R. Ultrasensitive mass sensing using mode localization in coupled micro-cantilevers. Appl. Phys. Lett. 2006, 88, 254102. [CrossRef]

57. Spletzer, M.; Raman, A.; Sumali, H.; Sullivan, J.P. Highly sensitive mass detection and identification using vibration localization in coupled micro cantilever arrays. Appl. Phys. Lett. 2008, 92, 114102. [CrossRef]

58. Hajhashemi, M.; Rasouli, A.; Bahreyni, B.; Member, S. Improving Sensitivity of Resonant Sensor Systems Through Strong Mechanical Coupling. IEEE J. Microelectromech. Syst. 2016, 25, 52-59. [CrossRef]

59. DeMartini, B.E.; Rhoads, J.F.; Zielke, M.A. A single input-single output coupled microresonator array for the detection and identification of multiple analytes. Appl. Phys. Lett. 2008, 93, 54102. [CrossRef]

60. Kang, H.; Yang, J.; Chang, H. A mode-localized accelerometer based on four degree-of-freedom weakly coupled resonators. In Proceedings of the 2018 IEEE Micro Electro Mechanical Systems (MEMS), Belfast, UK, 21-25 January 2018; pp. 960-963. [CrossRef]

61. Ruan, B.; Hao, Y.; Kang, H.; Shen, Q.; Chang, H. A Mode localized Tilt Sensor with Resolution of 2.4e-5 Degrees within the Range of 50 Degrees. In Proceedings of the INERTIAL 2020-7th IEEE International Symposium on Inertial Sensors and Systems, Proceedings, Hiroshima, Japan, 23-26 March 2020; pp. 1-4. [CrossRef]

62. Zhang, H.; Yuan, W.; Huang, J.; Li, B.; Chang, H. A high-sensitive resonant electrometer based on mode localization of the weakly coupled resonators. In Proceedings of the International Conference on Micro Electro Mechanical Systems 2016, Shanghai, China, 24-28 January 2016; pp. 87-90. [CrossRef]

63. Zhang, H.; Huang, J.; Yuan, W.; Chang, H. A high-sensitivity micromechanical electrometer based on mode localization of two degree of-freedom weakly coupled resonators. J. Microelectromech. Syst. 2016, 25, 937-946. [CrossRef]

64. Zhang, H.M.; Yuan, W.Z.; Li, B.Y.; Hao, Y.C.; Kraft, M.; Chang, H.L. A novel resonant accelerometer based on mode localization of weakly coupled resonators. In Proceedings of the International Conference on Solid State Sensors Actuators and Microsystems, Anchorage, AL, USA, 21-25 June 2015; pp. 1073-1076. [CrossRef]

65. Zhang, H.; Li, B.; Yuan, W.; Kraft, M.; Chang, H. An acceleration sensing method based on the mode localization of weakly coupled resonators. J. Microelectromech. Syst. 2016, 25, 286-296. [CrossRef]

66. Kang, H.; Yang, J.; Chang, H. A Closed-Loop Accelerometer Based on Three Degree-of-Freedom Weakly Coupled Resonator with Self-Elimination of Feedthrough Signal. IEEE Sens. J. 2018, 18, 3960-3967. [CrossRef]

67. Zhang, H.; Kang, H.; Chang, H. Suppression on Nonlinearity of Mode-Localized Sensors Using Algebraic Summation of Amplitude Ratios as the Output Metric. IEEE Sens. J. 2018, 18, 7802-7809. [CrossRef]

68. Rabenimanana, T.; Walter, V.; Kacem, N.; Le Moal, P.; Bourbon, G.; Lardies, J. Mass sensor using mode localization in two weakly coupled MEMS cantilevers with different lengths: Design and experimental model validation. Sens. Actuators A Phys. 2019, 295, 643-652. [CrossRef]

69. Chellasivalingam, M.; Pandit, M.; Kalberer, M.; Seshia, A. Ultra-fine Particulate Detection using Mode-localized MEMS Resonators. In Proceedings of the IFCS/EFTF 2019-Joint Conference of the IEEE International Frequency Control Symposium and European Frequency and Time Forum, Proceedings 2019, Orlando, FL, USA, 14-18 April 2019; pp. 1-5. [CrossRef]

70. Pandit, M.; Zhao, C.; Sobreviela, G.; Zou, X.; Seshia, A. A High Resolution Differential Mode-Localized MEMS Accelerometer. J. Microelectromech. Syst. 2019, 28, 782-789. [CrossRef]

71. Yang, J.; Zhong, J.; Chang, H. A Closed-Loop Mode-Localized Accelerometer. J. Microelectromech. Syst. 2018, $27,210-217$. [CrossRef]

72. Zhang, H.; Chang, H.; Yuan, W. Characterization of forced localization of disordered weakly coupled micromechanical resonators. Microsyst. Nanoeng. 2017, 3, 17023. [CrossRef] [PubMed]

73. Pandit, M.; Zhao, C.; Sobreviela, G.; Du, S.; Zou, X.; Seshia, A. Utilizing Energy Localization in Weakly Coupled Nonlinear Resonators for Sensing Applications. J. Microelectromech. Syst. 2019, 28, 1-7. [CrossRef]

74. Thiruvenkatanathan, P.; Yan, J.; Woodhouse, J.; Seshia, A. Enhancing parametric sensitivity in electrically coupled MEMS resonators. J. Microelectromech. Syst. 2009, 18, 1077-1086. [CrossRef]

75. Thiruvenkatanathan, P.; Yan, J.; Woodhouse, J.; Aziz, A.; Seshia, A. Effects of mechanical and electrical coupling on the parametric sensitivity of mode localized sensors. In Proceedings of the IEEE International Ultrasonics Symposium Proceedings 2009, Rome, Italy, 20-23 September 2009; pp. 1183-1186. [CrossRef]

76. Pourkamali, S.; Ayazi, F. Electrically coupled mems band pass filters: Part II. Without coupling element. Sens. Actuators A Phys. 2005, 122, 317-325. [CrossRef]

77. Thiruvenkatanathan, P.; Yan, J.; Woodhouse, J.; Aziz, A.; Seshia, A. Ultrasensitive mode-localized mass sensor with electrically tunable parametric sensitivity. Appl. Phys. Lett. 2010, 96, 3562-3563. [CrossRef] 
78. Thiruvenkatanathan, P.; Seshia, A. Mode-Localized Displacement Sensing. J. Microelectromech. Syst. 2012, 21, 1016-1018. [CrossRef]

79. Thiruvenkatanathan, P.; Yan, J.; Seshia, A. Ultrasensitive mode-localized micromechanical electrometer. In Proceedings of the International Frequency Control Symposium 2010, Newport Beach, CA, USA, 1-4 June 2010; pp. 91-96. [CrossRef]

80. Lyu, M.; Zhao, J.; Kacem, N.; Tang, B.; Liu, P.; Song, J.; Zhong, H.; Huang, Y. Computational investigation of high-order mode localization in electrostatically coupled microbeams with distributed electrodes for high sensitivity mass sensing. Mech. Syst. Signal Process. 2021, 158, 107781. [CrossRef]

81. Wang, Y.; Zhao, C.; Wang, C.; Cerica, D.; Baijot, M.; Xiao, Q.; Stoukatch, S.; Kraft, M. A mass sensor based on 3-DOF mode localized coupled resonator under atmospheric pressure. Sens. Actuators A 2018, 279, 254-262. [CrossRef]

82. Wang, Y.; Wang, C.; Zhao, C.; Liu, H.; Cerica, D.; Baijot, M.; Dupont, F.; Stoukatch, S.; Fraft, M. A Novel Qcm Mass Sensing System Incorporated with A 3-Dof Mode Localized Coupled Resonator Stiffness Sensor. In Proceedings of the 2019 20th Int. Conf. Solid-State Sensors, Actuators Microsystems Eurosensors XXXIII, Transducers 2019 Eurosensors XXXIII 2019, Berlin, Germany, 23-27 June 2019; pp. 1823-1826. [CrossRef]

83. Wang, Y.; Liu, H.; Wang, C.; Zhao, C.; Kraft, M. Towards a hybrid mass sensing system by combining a qcm mass sensor with a 3-dof mode localized coupled resonator stiffness sensor. IEEE Sens. J. 2021, 21, 8988-8997. [CrossRef]

84. Zhao, C.; Wood, G.S.; Xie, J.; Chang, H.; Pu, S.H.; Chong, H.M.; Kraft, M. A sensor for stiffness change sensing based on three weakly coupled resonators with enhanced sensitivity. In Proceedings of the IEEE International Conference on Micro Electro Mechanical Systems (MEMS) 2015, Estoril, Portugal, 18-22 January 2015; pp. 881-884. [CrossRef]

85. Zhao, C.; Wood, G.S.; Xie, J.; Chang, H.; Pu, S.; Kraft, M. A three degree-of-freedom weakly coupled resonator sensor with enhanced stiffness sensitivity. J. Microelectromech. Syst. 2016, 25, 38-51. [CrossRef]

86. Zhao, C.; Wood, G.S.; Xie, J.; Chang, H.; Pu, S.; Kraft, M. A comparative study of output metrics for a MEMS resonant sensor consisting of three weakly coupled resonators. J. Microelectromech. Syst. 2016, 25, 626-636. [CrossRef]

87. Zhao, C.; Wood, G.S.; Xie, J.; Chang, H.; Pu, S.; Kraft, M. A force sensor based on three weakly coupled resonators with ultrahigh sensitivity. Sens. Actuators A-Phys. 2015, 232, 151-162. [CrossRef]

88. Zhao, C.; Pandit, M.; Sobreviela, G.; Mustafazade, A.; Du, S.; Seshia, A. On the Noise Optimization of Resonant MEMS Sensors Utilizing Vibration Mode Localization. Appl. Phys. Lett. 2018, 112, 194103. [CrossRef]

89. Pai, P.; Pourzand, H.; Tabib-Azar, M. Magnetically coupled resonators for rate integrating gyroscopes. In Proceedings of the IEEE SENSORS, Valencia, Spain, 2-5 November 2014; pp. 1173-1176. [CrossRef]

90. Thiruvenkatanathan, P.; Yan, J.; Seshia, A. Common mode rejection in electrically coupled MEMS resonators utilizing mode localization for sensor application. In Proceedings of the International Frequency Control Symposium 2009, Besancon, France, 20 April 2009; pp. 358-363. [CrossRef]

91. Zhang, H.; Zhong, J.; Yuan, W.; Yang, J.; Chang, H. Ambient pressure drift rejection of mode-localized resonant sensors. In Proceedings of the IEEE International Conference on Micro Electro Mechanical Systems (MEMS) 2017, Las Vegas, NA, USA, 22-26 January 2017; pp. 1095-1098. [CrossRef]

92. Zhong, J.; Yang, J.; Chang, H. The temperature drift suppression of mode-localized resonant sensors. In Proceedings of the IEEE International Conference on Micro Electro Mechanical Systems (MEMS) 2018, Belfast, UK, 21-25 January 2018; pp. 467-470. [CrossRef]

93. Pandit, M.; Zhao, C.; Sobreviela, G.; Seshia, A. Immunity to Temperature Fluctuations in Weakly Coupled MEMS Resonators. In Proceedings of the 2018 IEEE SENSORS, New Delhi, India, 28-31 October 2018; pp. 1-4. [CrossRef]

94. Kang, H.; Ruan, B.; Hao, Y.; Chang, H. A Mode-Localized Resonant Accelerometer with Self-Temperature Drift Suppression. IEEE Sens. J. 2020, 20, 12154-12165. [CrossRef]

95. Pandit, M.; Zhao, C.; Sobreviela, G.; Seshia, A. Practical Limits to Common Mode Rejection in Mode Localized Weakly Coupled Resonators. IEEE Sens. J. 2020, 20, 6818-6825. [CrossRef]

96. Juillard, J.; Prache, P.; Ferreira, P.M.; Barniol, N. Impact of output metric on the resolution of mode-localized MEMS resonant sensors. In Proceedings of the 2017 Joint Conference of the European Frequency and Time Forum and IEEE International Frequency Control Symposium (EFTF/IFC) 2017, Besancon, France, 9-13 July 2017; pp. 506-509. [CrossRef]

97. Juillard, J.; Prache, P.; Ferreira, P.M.; Barniol, N. Ultimate limits of differential resonant MEMS sensors based on two coupled linear resonators. IEEE Trans. Ultrason. Ferroelectr. Freq. Control 2018, 65, 2440-2448. [CrossRef] [PubMed]

98. Thiruvenkatanathan, P.; Woodhouse, J.; Yan, J.; Seshia, A. Limits to mode-localized sensing using micro- and nanomechanical resonator arrays. J. Appl. Phys. 2011, 109, 104903. [CrossRef]

99. Zhang, Z.; Chang, H. Resolution limit of mode-localised sensors. Sci. China Inf. Sci. 2021, 64, 1-10.

100. Yang, J.; Kang, H.; Chang, H. A micro resonant electrometer with 9-electron charge resolution in room temperature. In Proceedings of the 2018 IEEE Micro Electro Mechanical Systems (MEMS) 2018, Belfast, UK, 21-25 January 2018; pp. 67-70. [CrossRef]

101. Kang, H.; Ruan, B.; Hao, Y.; Chang, H. A micromachined electrometer with room temperature resolution of $0.256 \mathrm{e} / \sqrt{ } \mathrm{Hz}$. IEEE Sens. J. 2020, 20, 95-101. [CrossRef]

102. Pandit, M.; Zhao, C.; Sobreviela, G.; Mustafazade, A.; Du, S.; Zou, X.; Seshia, A.A. Closed-Loop Characterization of Noise and Stability in a Mode-Localized Resonant MEMS Sensor. IEEE Trans. Ultrason. Ferroelectr. Freq. Control 2019, 66, 170-180. [CrossRef] 
103. Liang, J.; Hao, Y.; Kang, H.; Ruan, B.; Chang, H. A Mode-Localized Voltmeter with Resolution of 46.8 Nanovolts. In Proceedings of the 2019 20th International Conference on Solid-State Sensors, Actuators and Microsystems and Eurosensors XXXIII, TRANSDUCERS and EUROSENSORS XXXIII 2019, Berlin, Germany, 23-27 June 2019; pp. 226-229. [CrossRef]

104. Peng, B.; Hu, K.; Shao, L.; Yan, H.; Li, L.; Wei, X.; Zhang, W. A Sensitivity Tunable Accelerometer Based on Series-Parallel Electromechanically Coupled Resonators Using Mode Localization. J. Microelectromech. Syst. 2020, 29, 3-13. [CrossRef]

105. Stassi, S.; Laurentis, G.D.; Chakraborty, D.; Bejtka, K.; Chiodoni, A.; Sader, J.E.; Ricciardi, C. Large-scale parallelization of nanomechanical mass spectrometry with weakly-coupled resonators. Nat. Commun. 2019, 10, 3647. [CrossRef]

106. Pachkawade, V. State-of-the-Art in Mode-Localized MEMS Coupled Resonant Sensors: A Comprehensive Review. IEEE Sens. J. 2021, 21, 8751-8779. [CrossRef]

107. Malar, C.; Brian, G.; Adam, B.; Ashwin, A.S. Mass Tuning in Weakly Coupled Low-Q Piezoelectric MEMS Resonator Arrays for Particulate Sensing. In Proceedings of the 2020 IEEE 33rd International Conference on Micro Electro Mechanical Systems (MEMS) 2020, Vancouver, BC, USA, 18-22 January 2020; pp. 761-764. [CrossRef]

108. Manav, M.; Phani, A.S.; Cretu, E. Mode Localization and Sensitivity in Weakly Coupled Resonators. IEEE Sens. J. 2019, 19, 2999-3007. [CrossRef]

109. Tabrizian, R.; Ayazi, F. Acoustically-Engineered Multi-Port AlN-on-Silicon Resonators for Accurate Temperature Sensing. In Proceedings of the IEEE International Electron Devices Meeting 2013, Washington, DC, USA, 9-11 December 2013. [CrossRef]

110. Tabrizian, R. Temperature-Compensated Silicon-Based Bulk Acoustic Resonators. Ph.D. Thesis, Georgia Institute of Technology, Atlanta, GA, USA, 2013. Available online: https://smartech.gatech.edu/bitstream/handle/1853/52929/TABRIZIANDISSERTATION-2013.pdf (accessed on 27 October 2021).

111. Tabrizian, R.; Ayazi, F. Dual-Mode Vertical Membrane Resonant Pressure Sensor. In Proceedings of the MEMS 2014, San Francisco, CA, USA, 26-30 January 2014; pp. 120-123. [CrossRef]

112. Schodowski, S. Resonator self-temperature-sensing using a dual-harmonic-mode crystal oscillator. In Proceedings of the Annual Symposium on Frequency Control 1989, Denver, CO, USA, 31 May-2 June 1989; pp. 2-7. [CrossRef]

113. Campanella, H.; Narducci, M.; Merugu, S.; Singh, N. Dual MEMS Resonator Structure for Temperature Sensor Applications. IEEE Trans. Electron Devices 2017, 64, 3368-3377. [CrossRef]

114. Dalal., M.J.; Fu, J.L.; Ayazi, F. Simultaneous Dual-Mode Excitation of Piezo-on-Silicon Micromechanical Oscillator for SelfTemperature Sensing. In Proceedings of the International Conference on Micro Electro Mechanical Systems 2011, Cancun, Mexico, 23-27 January 2011; pp. 489-492. [CrossRef]

115. Fu, J.; Tabrizian, R.; Ayazi, F. Dual-Mode AlN-on-Silicon Micromechanical Resonators for Temperature Sensing. IEEE Trans. Electron. Devices 2014, 61, 591-598. [CrossRef]

116. Garcia-Gancedo, L.; Pedros, J.; Zhao, X.B.; Ashley, G.M.; Flewitt, A.J.; Milne, W.I.; Ford, C.J.B.; Lu, J.R.; Luo, J.K. Dual-mode thin film bulk acoustic wave resonators for parallel sensing of temperature and mass loading. Biosens. Bioelectron. 2012, 38, 369-374. [CrossRef]

117. Gu, C.C.; Xuan, W.P.; Dong, S.R.; Wang, X.Z.; Li, H.L.; Yu, L.Y.; Luo, J.K. Temperature calibrated on-chip dual-mode film bulk acoustic resonator pressure sensor with a sealed back-trench cavity. J. Micromech. Microeng 2018, 28, 075010. [CrossRef]

118. Li, Y.; Wang, J.; Luo, Z.; Chen, D.; Chen, J. A Resonant Pressure Microsensor Capable of Self-Temperature Compensation. Sensors 2015, 15, 10048-10058. [CrossRef]

119. Xie, Q.; Wang, N.; Sun, C.; Randles, A.; Singh, P.; Zhang, X.; Gu, Y. A Passively Temperature-Compensated Dual-Frequency AlN-On-Silicon Resonator for Accurate Pressure Sensing. In Proceedings of the International Conference on Micro Electro Mechanical Systems 2017, Las Vegas, NV, USA, 22-26 January 2017; pp. 977-981. [CrossRef]

120. Roshan, M.; Zaliasl, S.; Joo, K.; Souri, K.; Palwai, R.; Chen, L.; Singh, A.; Pamarti, S.; Miller, N.; Doll, J.; et al. A MEMS-Assisted Temperature Sensor With 20- $\mu \mathrm{K}$ Resolution, Conversion Rate of $200 \mathrm{~S} / \mathrm{s}$, and FOM of $0.04 \mathrm{pJK}^{2}$. IEEE J. Solid-State Circuits 2017, 52, 185-197. [CrossRef]

121. Ng, E.J.; Lee, H.K.; Ahn, C.H.; Melamud, R.; Kenny, T.W. Stability of silicon microelectromechanical systems resonant thermometers. IEEE Sens. J. 2013, 13, 987-993. [CrossRef]

122. Vig, J.R. Dual-mode oscillators for clocks and sensors. In Proceedings of the International Ultrasonics Symposium 1999, Lake Tahoe, NV, USA, 17-20 October 1999; pp. 859-868. [CrossRef]

123. Zhao, C.; Montaseri, M.; Wood, G.; Pu, S.; Seshia, A.; Kraft, M. A review on coupled MEMS resonators for sensing applications utilizing mode localization. Sens. Actuators A 2016, 249, 93-111. [CrossRef]

124. Wang, K.; Nguye, C.C. High-order medium frequency micromechanical electronic filters. J. Microelectromech. Syst. 1999, 8 , 534-556. [CrossRef]

125. Hung, L. High-Q Low-Impedance MEMS Resonators. Ph.D. Thesis, Electrical Engineering and Computer Sciences University of California, Berkeley, CA, USA, 2012. Available online: https:/ / www2.eecs.berkeley.edu/Pubs/TechRpts/2012/EECS-2012-218 .pdf (accessed on 1 December 2012).

126. Motiee, M. MEMS IF/RF Filters. Master's Thesis, Department of Mechanical Engineering, University of Waterloo, Waterloo, ON, Canada, 2003.

127. Wang, K.; Wong, A.C.; Nguyen, C.T.C. VHF free-free beam high-Q micromechanical resonators. J. Microelectromech. Syst. 2000, 9 , 347-360. [CrossRef] 
128. Johnson, R.A. Mechanical Filters in Electronics; Wiley: New York, NY, USA, 1983; Available online: https:/ / openlibrary.org/books / OL3491136M/Mechanical_filters_in_electronics (accessed on 26 August 1998).

129. Weinstein, D.; Bhave, S.; Tada, M.; Mitarai, S.; Morita, S. Mechanical Coupling of 2D Resonator Arrays for MEMS Filter Applications. In Proceedings of the IEEE International Frequency Control Symposium 2007, Geneva, Switzerland, 29 May-1 June 2007; pp. 1362-1365. [CrossRef]

130. Chandrahalim, H.; Bhave, S. Digitally-tunable MEMS filter using mechanically coupled resonator array. In Proceedings of the International Conference on Micro Electro Mechanical Systems 2008, Tucson, AZ, USA, 13-17 January 2008; pp. 1020-1023. [CrossRef]

131. Lopez, J.; Verd, J.; Uranga, A.; Murillo, G.; Giner, J.; MarigÃ, E.; Torres, F.; Abadal, G.; Barniol, N. VHF band-pass filter based on a single CMOS-MEMS double ended tuning fork resonator. Procedia Chem. 2009, 1, 1131-1134. [CrossRef]

132. Motiee, M.; Mansour, R.R.; Khajepour, A. Novel MEMS filters for on-chip transceiver architecture, modeling and experiments. J. Micromech. Microeng. 2006, 16, 407-418. [CrossRef]

133. Giessibl, F.J. A direct method to calculate tip sample forces from frequency shifts in frequency modulation atomic force microscopy. Appl. Phys. Lett. 2001, 78, 123-125. [CrossRef]

134. Seshia, A.; Palaniapan, M.; Roessig, T.; Howe, R.; Gooch, R.; Schimert, T.; Montague, S. A vacuum packaged surface micromachined resonant accelerometer. J. Microelectromech. Syst. 2002, 11, 784-793. [CrossRef]

135. Leeson, D.B. A simple model of feedback oscillator noise. Proc. IEEE 1966, 54, 329-330. [CrossRef]

136. Zhao, C.; Pandit, M.; Sobreviela, G.; Mustafazade, A.; Du, S.; Zou, X.; Seshia, A. A Direct Feedback Oscillator Topology Employing Weakly Coupled Resonators for Gain Control. In Proceedings of the IEEE International Frequency Control Symposium (IFCS) 2018, Olympic Valley, CA, USA, 21-24 May 2018. [CrossRef] 